\title{
A comprehensive single-cell map of T cell exhaustion-associated immune environments in human breast cancer
}

\section{Sandra Tietscher}

University of Zurich

Johanna Wagner

DKFZ and NCT https://orcid.org/0000-0002-5087-3657

\section{Tobias Anzeneder}

PATH Biobank

Claus Langwieder

Pathologie Josefshaus

\section{Martin Rees}

Pathologie Josefshaus

\section{Bettina Sobottka}

University Hospital Zurich

\section{Natalie de Souza}

University of Zurich

Bernd Bodenmiller ( $\sim$ bernd.bodenmiller@imls.uzh.ch )

University of Zürich, https://orcid.org/0000-0002-6325-7861

\section{Article}

Keywords: Breast cancer, T cell exhaustion, tumor immune microenvironment, Luminal B, single-cell analysis, multiplexed imaging

Posted Date: January 5th, 2022

DOI: https://doi.org/10.21203/rs.3.rs-1155834/v1

License: (c) (i) This work is licensed under a Creative Commons Attribution 4.0 International License.

Read Full License 


\section{A comprehensive single-cell map of T cell exhaustion-associated 2 immune environments in human breast cancer}

4 Sandra Tietscher ${ }^{1,2,3}$, Johanna Wagner ${ }^{1,4}$, Tobias Anzeneder ${ }^{5}$, Claus Langwieder ${ }^{6}$, Martin Rees $^{6}$, Bettina Sobottka ${ }^{7}$, Natalie de Souza ${ }^{1,2}$ and Bernd Bodenmiller ${ }^{1,2^{*}}$

1. Department of Quantitative Biomedicine, University of Zurich, Zurich, Switzerland

2. Institute for Molecular Health Sciences, ETH Zurich, Zurich, Switzerland

3. Life Science Zurich Graduate School, ETH Zurich and University of Zurich, Zurich, Switzerland

4. Department of Translational Medical Oncology, German Cancer Research Center (DKFZ) and National Center for Tumor Diseases (NCT) Heidelberg, Heidelberg, Germany

5. Patients' Tumor Bank of Hope (PATH), Munich, Germany

6. Pathology at Josefshaus, Dortmund, Germany

7. Department of Pathology and Molecular Pathology, University Hospital Zurich, Zurich and University of Zurich, Switzerland

${ }^{*}$ correspondence to bernd.bodenmiller@uzh.ch 


\section{ABSTRACT}

24 Immune checkpoint therapy in breast cancer remains restricted to triple negative patients, and long-term clinical benefit is rare. The primary aim of immune checkpoint blockade is to prevent or reverse exhausted $\mathrm{T}$ cell states, but the causes and implications of $\mathrm{T}$ cell exhaustion in breast tumors are not well understood. Here, we used single-cell transcriptomics combined

28 with imaging mass cytometry to comprehensively study exhausted and non-exhausted immune environments in human breast tumors, with a focus on Luminal subtypes. We found

30 that the presence of a PD-1 $1^{\text {high }}$ exhaustion-like $\mathrm{T}$ cell phenotype was indicative of an inflammatory immune environment with a characteristic cytotoxic profile and spatial features.

32 Accumulation of natural killer $T$ cells and increased myeloid cell activation in exhausted immune environments provide further support for tissue inflammation in these environments.

34 Consistent with this, our comprehensive map of cellular interactions within the breast tumor microenvironment revealed elevated immunomodulatory, chemotactic, and cytokine signaling in exhausted environments. These data reveal fundamental differences between exhausted and non-exhausted immune environments within Luminal breast cancer, and show that expression of PD-1 and CXCL13 on T cells, and MHC-I - but not PD-L1 - on tumor cells are strong distinguishing features between these environments; these factors are potential new biomarkers for patient stratification.

\section{KEYWORDS}

Breast cancer, $T$ cell exhaustion, tumor immune microenvironment, Luminal $B$, single-cell analysis, multiplexed imaging 
Immune checkpoint blockade therapies have improved patient outcomes in many human cancer types ${ }^{1-4}$. Breast cancer is an exception, previously believed to be due to poor immunogenicity ${ }^{5}$, but several studies have identified a strong influence of the immune infiltrate on breast cancer progression ${ }^{6,7}$. T cell infiltration in particular impacts patient survival for all breast cancer subtypes, but the effect on prognosis may be positive or negative depending on 54 the subtype ${ }^{8}$, highlighting the complexity of the tumor-immune interaction. Recently, atezolizumab and pembrolizumab, two checkpoint inhibitors targeting the PD-1/PD-L1 pathway, have been approved for patients with triple-negative breast cancer ${ }^{9}$. These treatments increase progression-free survival by several months, but most patients do not gain

58 long-term clinical benefit, and no approved immunotherapeutic options currently exist for patients with other breast cancer subtypes. Our understanding of the mechanisms of resistance or response to immunotherapy is incomplete, as is our knowledge of the complex cellular interactions in the tumor immune microenvironment (TIME). In order to design new immunotherapies and to effectively use existing ones for luminal breast cancer patients, it is critical to understand the TIME as a whole.

64 T cells make up a large part of the immune infiltrate in most solid tumors, including breast tumors $^{8}$, and provide an important line of defense against cancer cells. Upon activation, cytotoxic T lymphocytes (CTLs) can exert their effector function through cytolytic molecules such as granzymes and granulysin or via inflammatory cytokines such as IFN-y and TNF$\alpha^{10,11}$. At sites of chronic inflammation - such as tumors - where the elimination of target cells fails and T cell receptor (TCR) signaling persists, T cells may become "exhausted", a cellular state characterized by an elevated co-expression of inhibitory checkpoint receptors such as PD-1, CTLA-4, Tim-3, and LAG-3 ${ }^{12}$. Prevention or reversal of T cell exhaustion - also termed $T$ cell dysfunction - is the primary aim of most immune checkpoint blockade therapies ${ }^{12}$. Originally discovered under conditions of viral infection, $\mathrm{T}$ cell exhaustion is associated with reduced effector functions. Although loss of proliferative potential was thought to be a characteristic of exhausted T cells, recent evidence suggests that they may still proliferate in human tumors ${ }^{13,14}$ and that $T$ cells with an exhausted phenotype expand after anti-PD1 treatment in breast tumors ${ }^{15}$, but the extent to which these cells retain their anti-tumor functions is unclear ${ }^{16}$.

Myeloid cells are also present in the immune infiltrate in most breast tumors, with pro- and anti-tumor effects reported ${ }^{17}$. Tumor-associated macrophages (TAMs) can directly suppress $\mathrm{T}$ cell responses by expression of PD-L1 and other immunomodulatory molecules, or indirectly via recruitment of regulatory $T$ cells $\left(T_{\text {regs }}\right.$ ). Dendritic cells (DCs) prime anti-tumor $T$ cell function by surface-presentation of tumor-derived antigens ${ }^{18}$. Myeloid cells may also promote angiogenesis and tissue remodeling ${ }^{19}$, and other immune cell types like $B$ cells and natural killer (NK) cells further add to the complexity of immune-mediated effects on tumors.

Recent technological developments allow for increasingly robust and comprehensive singlecell analysis of tumor microenvironments at the transcriptional and protein levels ${ }^{20-22}$, facilitating the investigation of cell type abundances, cellular phenotypes, and cell-to-cell communication. Spatially resolved multiplexed methods further enable the study of cellular neighborhoods and spatially defined tissue patterns such as tertiary lymphoid structures (TLS) in cancer $^{23-26}$. Although transcriptomic studies have provided insight into the immune 
composition of both treatment naïve and anti-PD1 treated breast tumors at the single-cell leve ${ }^{15,27,28}$, a systematic analysis of exhausted and non-exhausted TIMEs and a clinically

94 established TIME-based classification system for patient stratification are missing. In previous work, we used mass cytometry-based cellular phenotyping to classify (mainly luminal) breast 96 tumors into three groups based on infiltrating T cell and myeloid cell phenotypes ${ }^{29}$. One group showed strong enrichment of an exhaustion-like T cell phenotype. This group accounted for around $13 \%$ of all tumors and included both $\mathrm{ER}^{+}$and $E R^{-}$tumors and might be amenable to immune checkpoint inhibitor therapy. A second group, accounting for $48 \%$ of all tumors, also contained a high number of $T$ cells but did not display signs of immune exhaustion.

To deeply characterize the TIME in (luminal) breast cancer and to study which cell-to-cell interactions contribute to an exhausted TIME state, we used single-cell RNA sequencing (scRNA-seq) combined with imaging mass cytometry (IMC) to systematically analyse the

104 TIME of breast tumors with and without signs of $T$ cell exhaustion (called "exhausted" and "non-exhausted" environments hereafter). We discovered that T cells expressing exhaustion

106 markers display hallmarks of tumor reactivity and proliferation, and that their presence coincides with elevated MHC-I expression on tumor cells. High levels of expression of GZMB

108 and FASL (encoding Granzyme B and Fas ligand) but low levels of IFNG and TNF (encoding Interferon- $\gamma$ and TNF- $\alpha$ ) in these exhausted-like T cells are suggestive of an altered cytotoxic

110 profile but remaining capacity for tumor cell killing. CXCL13 was a common marker of PD$1^{\text {high }} \mathrm{CD} 8^{+}$and PD- $1^{\text {high }} \mathrm{CD} 4^{+} \mathrm{T}$ cells. We also found that sites of $\mathrm{B}$ cell and $\mathrm{CXCL} 13^{\text {high }} \mathrm{T}$ cell

112 accumulation, but not mature TLS, are more frequent in exhausted immune environments. Exhausted breast tumor environments were also enriched in cytotoxic CSF-1+ natural killer T

114 (NKT) cells and showed evidence of inflammation. Finally, we provide a comprehensive map of cellular interactions within the breast TIME, revealing strongly elevated immunomodulatory,

116 chemotactic, and cytokine signaling in tumors with an exhausted immune environment.

Our findings suggest that the fundamental differences between the exhausted and non118 exhausted immune environments in our study may be explained by different immune escape mechanisms - avoidance of tumor-specific T cell activation in non-exhausted environments 120 and progressive $\mathrm{T}$ cell dysfunction through chronic inflammatory signaling in exhausted environments. These differences are likely to affect clinical response to different 122 immunotherapies and may explain why PD-L1 blockade has shown limited success so far. Here, we describe exhaustion-associated TIME characteristics that might facilitate the 124 discovery of new therapeutic targets and propose PD-1, CXCL13 and MHC-I as a new biomarker combination for patient stratification.

\section{RESULTS}

\section{A single-cell map of immune environments in breast tumors}

In order to systematically study the TIME, we performed droplet-based scRNA-seq of 14 130 immune-infiltrated breast tumors (Fig. 1A, top), 12 of which were also included in the cohort analyzed by Wagner et al. ${ }^{29}$. We selected the tumors based on these previous mass cytometry data. Half of the samples contained exhausted $T$ cells (i.e., PD- $1^{\text {high }} / C T L A-4^{\text {high }} / C D 38^{\text {high }} T$ cells); we annotated these as immune environment 1 (IE1). The other half mainly contained T cells that did not express exhaustion markers and were annotated as immune environment 2 
(IE2). With one exception, all tumors analyzed by scRNA-seq were classified as luminal (S1A, left). Although all tumor grades were represented, most tumors were of grade 3 (S1A, right).

We subjected live, dissociated cells with no cell type enrichment to single-cell transcriptome

138 sequencing using the 10x Genomics platform. After standard data pre-processing, 119,000 high-quality cell measurements remained in the dataset (Fig. S1B), and no batch effect was

140 apparent for individual tumors (Fig. 1B) or immune environments (Fig. 1C). We manually annotated graph-based clusters (Fig. S1C) based on differential expression analysis and 142 known marker genes for the main expected cell types (Fig. 1D-1E, Fig. S1D). This revealed $36,000 \mathrm{~T}$ and NK cells, which clustered together due to their transcriptional similarity; 27,000

144 cells belonging to the myeloid lineage (monocytes, macrophages and DCs, excluding granulocytes); and 18,000 epithelial cells. Other cell types were present at lower frequencies.

146 We did not recover adipocytes and neutrophils, likely because they are particularly vulnerable to sample processing and cryopreservation damage ${ }^{30,31}$. We observed few significant 148 differences in frequencies of the main cell types between tumors with IE1 versus IE2 (Fig. 1F). Importantly, although different T cell phenotypes drove the distinction between IE1 and IE2,

150 the overall $\mathrm{T}$ cell frequency was similar in both groups.

To understand our scRNA-seq based findings in the in situ tumor context, we performed IMC 152 on formalin-fixed, paraffin embedded (FFPE) tissue of 12 of the 14 sequenced samples (Fig. $1 \mathrm{~A}$, bottom). For each sample, two consecutive FFPE sections were stained with two different

154 panels of antibodies and/or mRNA probes (Fig. S2A, Fig. S12, Fig. S13): Panel 1 (the Protein Panel) consists of 42 metal-labeled antibodies, and Panel 2 (the RNA Panel) includes 12 156 oligonucleotide probes ${ }^{24}$, the majority targeting cytokine mRNAs, and 26 antibodies. The marker choice for both panels was informed by analysis of the scRNA-seq data. Prior to IMC,

158 we used whole-slide immunofluorescence imaging of cell type markers to select four to six regions of interest (ROIs) representative of the general tissue structure. For samples that had

160 immature or mature TLS (defined as sites of B cell accumulation without a clear center or sites with dense round $\mathrm{B}$ cell accumulation, respectively ${ }^{32}$ ), additional regions including these 162 structures were imaged (Fig. S2B). In total, we acquired 77 ROls for each panel (Fig. 1G), and data pre-processing and single-cell segmentation resulted in more than 400,000 single cells

164 per panel, with manually matched ROls for consecutive slices having similar cell numbers (Fig. S2C). We performed graph-based clustering based on average marker expression to 166 identify the cell types present (Fig. 1H, Protein Panel: Fig. S2D-S2G, RNA Panel: Fig. S3A$\mathrm{S} 3 \mathrm{C}$ ). All cell types identified in the scRNA-seq dataset as well as neutrophils were identified

168 in our IMC dataset; neutrophils were generally rare but were present at slightly higher frequency in IE1 samples than in IE2 samples (Fig. S2H). For the RNA Panel IMC dataset, we

170 assigned a binary cytokine expression status to each cell based on the signal intensity for the given cytokine corrected by that of a background mRNA probe complementary to the bacterial

$172 D a p B$ mRNA. These data were then used for a comprehensive, spatially resolved, immunefocused analysis of the tumor microenvironment.

PD-1 ${ }^{\text {high }} /$ CTLA-4 ${ }^{\text {high }}$ T cells are tumor-reactive, proliferative, and coincide with high MHC- 
Expression of $\mathrm{T}$ cell exhaustion markers at the protein level is the most distinguishing feature of the two immune environments IE1 and IE2 in our study cohort. To further probe the T cell functional states in these tumors, we analyzed the transcriptomes of $T$ cell subtypes. We also 180 included NK cells in this analysis, since these cells have transcriptional and functional similarities with $\mathrm{T}$ cells and can reportedly undergo exhaustion ${ }^{33}$. We first used a pseudobulk 182 approach, averaging read counts across cells for individual patient samples. This identified several genes expressed at higher levels in IE1 than IE2, including transcripts PDCD1, 184 CD276, and HAVCR2 encoding immune checkpoint receptors PD-1, B7-H3, and Tim-3, respectively (Fig. 2A), as expected from our previous mass cytometry data ${ }^{29}$. A number of 186 genes normally associated with T cell activation, such as MKI67 (encoding Ki-67) and GZMB (encoding Granzyme B), were also expressed at higher levels in IE1 than IE2 (Fig. 2A). In 188 addition, mRNAs encoding three transcription factors (IRF4, BATF4, and TOX) previously associated with strong and/or chronic TCR signaling ${ }^{34-36}$ were expressed at significantly higher 190 levels in T and NK cells from IE1 than from IE2 (Fig. 2B). In contrast, TCF7, which encodes a transcription factor positively associated with favorable response to checkpoint therapy in 192 melanoma ${ }^{37}$, was elevated in IE2 compared to IE1. Finally, we found that cytokine and cytokine receptor expression was generally higher in T and NK cells from IE1 than from IE2, 194 with the most significant differences seen for CCL3, CXCR6, CSF1, and IL13, all of which have roles in inflammatory signaling (Fig. 2C). Running the same analysis while accounting 196 for tumor grade gave similar results (Fig. S11A).

To compare the phenotypic composition of the T and NK cell compartment in the IE1 and IE2 198 immune environments, we performed graph-based subclustering of the scRNA-seq data and annotated cellular subtypes based on differential expression analysis. This revealed $14 \mathrm{~T}$ cell 200 clusters, four NK cell clusters, and one NKT cell cluster (Fig. 2D-2F, Fig. S4A-C). We identified a $T_{\text {reg }}$ cluster as well as a naïve $T$ cell cluster based on canonical marker expression 202 (FOXP3/IL2RA and CCR7, respectively). In addition to a CD8 ${ }^{+}$exhausted T cell cluster (TCD8-exhausted) annotated based on expression of exhaustion markers (e.g., PDCD1 and 204 LAG3) and enriched in IE1 (Fig. 2E), we identified four CTL clusters: T-cytotoxic-1, 2, and 4 (Fig. S4D), were present in similar proportions in IE1 and IE2. T-cytotoxic-3 was significantly 206 more frequent in IE2 environments (Fig. 2E), and overexpressed early activation (CD69) and cytotoxicity-mediating mRNAs (TNF, GZMK, GZMA) in addition to mRNAs encoding a range 208 of ribosomal proteins and the main TCR components (CD3D, CD 3G, CD3E, TRAC), hinting at weak or transient TCR engagement of these cells ${ }^{38,39}$. We further identified two $C D 4^{\text {high }} \mathrm{T}$ 210 cell clusters enriched in IE1 that highly expressed PDCD1 along with known markers of $\mathrm{T}$ follicular helper (Tfh) cells (clusters Tfh-1 and Tfh-2) ${ }^{40}$. Finally, four T cell clusters (T-mixed-1

212 to 4) contained both CD4 and CD8 cells (Fig. S4E) and showed no IE-specific enrichment. We identified two more IE1-enriched clusters: an NKT cell cluster that co-expressed CD3E with 214 classical NK cell markers such as NCAM1, NCR1, and KLRC1 (Fig. 2E-2F, Fig. S4F) and a proliferating cell cluster (T-proliferating) that highly expressed MKI67 along other cell division

216 genes. Enrichment of individual clusters in IE1 or IE2 was not driven by differences in tumor grade (Fig. S11B). Our scRNA-seq analysis was largely confirmed by the IMC data, where we 218 also identified $T_{\text {regs }}$, NK cells, CD8 $/$ PD- $1^{\text {low }}, \mathrm{CD}^{+} / \mathrm{PD}-1^{\text {high }}, \mathrm{CD} 4^{+} / \mathrm{PD}-1^{\text {low }}$, and $\mathrm{CD} 4^{+} / \mathrm{PD}-1^{\text {high }}$ metaclusters (Fig. S5A-S5C), with $\mathrm{CD} 8^{+} / \mathrm{PD}-1^{\text {high }}$ and $\mathrm{CD} 4^{+} / \mathrm{PD}-1^{\text {high }} \mathrm{T}$ cells enriched in IE1 220 tumors (albeit not significantly when considering the IMC data alone, Fig S5C).

Next, we explored exhaustion-associated phenotypic $\mathrm{T}$ cell states in more detail. $\mathrm{T}$ cell exhaustion has been described as a continuous process during which self-renewing progenitor 
cells express the transcription factor TCF7, which is then gradually lost with increasing exhaustion and decreasing proliferation potential ${ }^{41}$. We indeed observed reduced expression of TCF7 mRNA in IE1 (Fig. 2B) and specifically in the IE1-enriched T-CD8-exhausted cluster 226 (Fig. 2E, 2G), although this could not be observed at the protein level in IMC (Fig. S5D). However, IE1 environments are also enriched in proliferating T cells compared to IE2 (Fig. 228 2E), and IMC analysis confirmed elevated Ki-67 expression in the PD-1 ${ }^{\text {high }}$ T cell subsets (Fig. S5E). Additionally, we found that the IE1-enriched T-CD8-exhausted cluster expressed higher 230 levels of many transcripts that distinguish tumor-reactive T cells from bystander T cells (which do not experience TCR signaling from the tumor and can be included in the naïve or cytotoxic 232 clusters) $)^{41,42}$ when compared to other $C D 8^{+}$T cell clusters (Fig. 2G). These include transcripts encoding CD39 (ENTPD1), CD103 (ITGAE), inhibitory receptors like PD-1 (PDCD1), LAG-3, 234 Tim-3 (HAVCR2), and CTLA-4, T cell activation markers such as 4-1BB (TNFRSF9) and GITR (TNFRSF18), and the B cell chemoattractant CXCL13. The T-proliferating cluster was also 236 enriched in all tumor reactivity- and exhaustion-related transcripts and was low in TCF7 in comparison to non-proliferating cells (all other clusters) (Fig. 2H). Taken together, our results indicate that tumor reactivity coincides with CTL exhaustion signatures and that tumor-reactive $T$ cells remained highly proliferative despite displaying characteristics of exhaustion.

240 We investigated potential sources of the difference in CD8+ T cell activation in IE1 and IE2. Pseudobulk analysis showed that levels of CD274 mRNA, which encodes the PD-1 ligand PD-

242 L1, were not significantly different between IE1 and IE2 either in the epithelial or in the myeloid subset (Fig. S4H-S4I), but revealed significantly higher expression of MHC-I-encoding genes

244 in epithelial cells of IE1-classified tumors compared to IE2-classified tumors (Fig. S4H). This was even more apparent in the IMC analysis, where we observed significantly higher 246 expression of HLA-ABC protein (the main MHC-I complex) on IE1 tumor cells (Fig. 2I). In contrast, HLA-ABC expression did not differ in high versus low-grade samples in our cohort

248 (Fig. S11C). Our data show that MHC class I expression constitutes a major difference between IE1 and IE2 tumor cells and show that, at least in this cohort, MHC-I is better 250 associated with CD8+ T cell activation than is PD-L1. Whole-slide IHC analysis of CD8+ T cell infiltration for 12 samples used in this study and 13 additional samples from the original

252 Wagner et al. cohort further showed that half of IE1 tumors, but none of the IE2 tumors, were classified as immune-infiltrated (Fig. S4J). This suggests an association of a "hot" immune phenotype with $T$ cell exhaustion, consistent with previous reports ${ }^{43,44}$.

Enrichment of antigen-experienced T cells in IE1 was not restricted to the CD8+ subset: The frequency of Tfh cells, an antigen-experienced PD- $1^{\text {high }} \mathrm{CD} 4^{+} \mathrm{T}$ cell type known to be involved in $\mathrm{B}$ cell maturation and differentiation ${ }^{45}$, was higher in IE1 (Fig. 2E) and strongly correlated

258 with the frequency of $\mathrm{CD}^{+}$exhausted T cells (Fig. S4K). Tfh frequency was also correlated with plasma cell but not B cell frequency (Fig. S4K), indicating that Th cells might act as 260 mediators of B cell differentiation into plasma cells in this context. In line with previous reports, both the T-CD8-exhausted and Tfh cell clusters in our dataset express CXCL13 (Fig. 2E-G),

262 and expression of CXCL13 and PDCD1 correlated across clusters and across patients (Fig. S4L and S5G). Elevated CXCL13 expression in IE1 was confirmed in IMC on both transcript and protein levels (Fig. 2J-2K) and the proportions of $\mathrm{CXCL} 13^{+} \mathrm{T}$ cells measured by scRNAseq and IMC on two separate pieces of the same tumor were highly correlated (Fig. S5F), 266 indicating that $\mathrm{CXCL} 13^{+} \mathrm{T}$ cell frequency is a tumor-wide characteristic. Our data suggest that $\mathrm{CXCL} 13^{+} \mathrm{T}$ cell frequency better distinguishes the IE1 and IE2 environments than frequency of PD-1 $1^{\text {high }} \mathrm{T}$ cells (Fig. $2 \mathrm{~K}$, compare Fig. S5C) and this difference could not be explained by 
tumor grade (Fig. S11D). Taken together, these findings indicate that T cells in the exhausted immune environment are proliferative, show a tumor-reactive signature and, despite signs of terminal $\mathrm{T}$ cell exhaustion, might retain more anti-tumor activity than $\mathrm{T}$ cells in tumors with a non-exhausted immune environment.

\section{Cytotoxic potential in exhausted immune environments is altered but not abolished}

Next, we examined how the two immune environments differ in T and NK cell cytotoxic 276 potential, focusing on the main molecules involved in CTL-mediated target cell killing. Pseudobulk comparison of nine selected genes in IE1 versus IE2 samples revealed that the

278 only cytotoxic molecule significantly elevated in the IE2 T and NK cell compartments was TNF, and that GZMB, GNLY (encoding Granulysin), and FASL (encoding Fas ligand) were 280 overexpressed in IE1 (Fig. 3A). GZMB was amongst the top differentially expressed genes (Fig. 2A), and we also observed a trend towards elevated Granzyme B protein expression in 282 CTLs from IE1 tumors with IMC (Fig. S6A). GZMB and FASL expression were highest in the T-CD8-exhausted cluster (Fig. 3B), indicating that these $T$ cells have the potential to exert

284 cytolytic effector functions despite their exhausted phenotype. Their cytotoxic profile is, however, different from that of the other CTL clusters, especially T-cytotoxic- 1 which highly expresses the classical cytotoxicity-related cytokines IFNG and TNF.

In order to assess whether the difference in cytotoxic potential between IE1 and IE2 can be better described in the context of a gradual exhaustion process, we placed the single-cells of our $\mathrm{CD}^{+} \mathrm{T}$ cell subsets on a linear pseudotime trajectory. We used a Bayesian approach 290 called Ouija ${ }^{46}$, which performs pseudotime ordering upon input from a given set of markers, in our case levels of transcripts associated with T cell activation or dysfunction (Fig. S6B). The 292 output was a trajectory with naïve T cells on one end and exhausted T cells on the other, with the cytotoxic clusters intermediate (Fig. 3C-3D). The clusters previously identified as 294 transition/early activation phenotypes (T-cytotoxic-3 and -4 , Fig. 2) had lower pseudotime scores than the other cytotoxic clusters, supporting the validity of our pseudotime ordering

296 (Fig. 3D). Not surprisingly, mean pseudotime scores were higher for IE1-classified samples (Fig. 3E), but there was substantial variability within the individual IE groups, supporting the

298 notion of immune exhaustion as a continuum and suggesting that the T cells in the IE1 samples are further along this continuum.

300 We examined gene expression profiles over pseudotime to identify early- and late-exhaustiongenes, associated with IE2 and IE1, respectively. We discovered that CXCL13, CTLA-4, and

$302 P D-1$ had the sharpest increases at the late exhausted stage (Fig. S6B). LAG-3 and TIGIT displayed a more gradual increase, which also began relatively early in the pseudotime 304 course. Among the cytotoxic genes, we observed an early increase of IFNG and TNF, closely followed by GZMB; but these three markers subsequently followed different trajectories (Fig.

306 3F). GNLY expression was significantly increased in IE1 versus IE2 in pseudobulk analysis (Fig. 3A) and had a pseudotime profile with a peak in the middle and a decrease in the late 308 stages. The main source of GNLY expression within the T and NK cell compartments were NKT cells (Fig. 3B, S4F), a strongly cytotoxic cell type with a poorly understood role in human cancer $^{47}$. 
To better understand how NKT cells might act on the IE1 environment, we examined their expression profile in more detail. One of the few uniquely overexpressed genes in the NKT cell cluster was CSF1 (Fig. 3G), which encodes a cytokine important for the activation and 314 differentiation of myeloid cells ${ }^{48}$. We used this marker to annotate NKT cells in our IMC dataset, defining CSF $-1^{+} \mathrm{T}$ and NK cells as NKT cells and confirming the overrepresentation of this cell type in IE1 environments at the protein level (Fig. S6C).

Thus, the main effectors of T and NK cell-mediated cytotoxicity appear to be distinct in the two 318 immune environments. Our data indicate that the T cells in IE1 and IE2 samples are present in an exhaustion continuum, and that IE1 T cells are further along this continuum than those 320 in IE2. The exhausted-like immune environment IE1 is characterized by strong upregulation of GZMB in exhausted T cells and the presence of GNLY-expressing NKT cells, suggesting 322 cytotoxic potential. The previously unreported expression of CSF-1 by NKT cells in exhausted immune environments may represent a link between $T$ cell activity and myeloid-cell mediated 324 immune responses.

\section{Myeloid cells in exhausted immune environments indicate increased inflammation}

We went on to explore in more detail how different myeloid cell subsets might affect $\mathrm{T}$ cell 328 state and disease progression in IE1 and IE2. We first performed pseudobulk analysis on the myeloid cell fraction (monocytes, macrophages and DCs, excluding granulocytes) to compare 330 the two IEs (Fig. 4A). Several cytokine-encoding mRNAs were overexpressed in IE1 (Fig. S7A), most notably CCL18, which has previously been associated with breast cancer

332 metastasis ${ }^{49}$. We also observed overexpression of genes encoding major complement system components in IE1 compared to IE2 (Fig. S7A), indicating higher inflammatory and phagocytic

334 potential of IE1 myeloid cells. In IE2-classified tumors, we observed upregulation of CD55 and CD46, which encode proteins that limit complement function ${ }^{50}$, in $\mathrm{T}$ and NK cells (Fig. S7B).

336 Finally, there was increased expression of transcripts encoding matrix metalloproteinases, implicated in breast cancer invasion and metastasis ${ }^{51}$, and of metallothioneins, which protect

338 cells from oxidative stress and cytotoxicity, in IE1 myeloid cells (Fig. S7A). Running the same analysis while accounting for tumor grade revealed a similar trend (Fig. S11E). Taken

340 together, these data are indicative of inflammation in IE1 tumors.

Graph-based subclustering of the scRNA-seq profiles from the myeloid cell fraction revealed

342 two clusters of monocytes or early differentiating macrophages (mono-1 and mono-2), seven clusters of TAMs, five clusters of DCs, and one cluster of proliferating myeloid cells (Fig. 4B,

344 Fig. S7C). All patient samples contained monocytes, TAMs, and DCs (Fig. S7D), although three out of the seven TAM clusters were found mainly in one patient sample each (Fig. S7E).

346 Most monocyte and TAM clusters tended to be more frequent in IE1 tumors without any individual cluster reaching significance (Fig. 4C), whereas IE2 tumors showed enrichment of 348 one specific TAM cluster (cluster TAM-2) as well as classical DCs type 2 (cluster cDC2). Enrichment of CDC2 in IE2 was not driven by differences in tumor grade (Fig. S11F).

350 A closer survey of differentially expressed genes in the identified subclusters revealed a monocyte-specific gene signature for cells of the mono- 1 cluster. The mono-2 cells showed

352 patterns also overlapping with several TAM clusters and likely represent a transition phenotype from monocyte to mature macrophage (Fig. 4D). TAM clusters showed high 
354 expression of $C D 68, C D 163, M R C 1$ (also known as CD206), and MSR1 (also known as CD204) (Fig. S7F). TAM-1 and TAM-2 cluster phenotypes were similar, both highly expressed

356 cytokine-encoding mRNAs such as IL10, CCL3, CCL4, and CXCL8 with the TAM-1 cluster cells additionally high in TNF. Conversely, the TAM-3 cluster was unique in its high expression 358 of mRNAs encoding T cell-attracting chemokines CXCL9, CXCL10, and CXCL11 as well as a range of interferon-induced genes. TAM-4 and TAM-5 clusters were fully mature macrophage 360 subsets, co-expressing MRC1, MSR1, and SIGLEC1 (also known as CD169) but comparatively low in cytokine expression. Finally, TAM-6 and TAM-7 clusters were both 362 dominated by cells from the same patient (TBB338) and each displayed a unique transcriptional profile.

364 Having observed exhausted T cells and signs of inflammation and myeloid cell differentiation, we next examined crosstalk of the different immune compartments. We quantified $\mathrm{T}$ cell366 attractive and T cell-suppressive properties of myeloid cells using gene signatures that we assembled from the literature (Genes: Supplementary Table S5). We were able to functionally 368 validate the $T$ cell attraction signature and a subset of markers within the $T$ cell suppression signature using spatial IMC analysis (Fig S7G-S7H). The two signatures showed a positive 370 correlation at the single-cell level (Fig. S7I), at the patient level (Fig. S7J), and at the level of phenotypic clusters (Fig. 4E), suggesting that myeloid cells that harbor a strong suppressive 372 potential might also actively attract T cells. Most TAM clusters showed high scores for both signatures, whereas monocyte and DC clusters tended to have low scores. Both signatures 374 tended to be enriched in IE1 tumors compared to IE2 tumors, as were gene signatures for "classically activated" (M1) and "alternatively activated" M2 macrophages ${ }^{27}$ (Fig. S7K-S7L).

376 This confirmed an overall higher activation and differentiation state of myeloid cells in IE1 environments than in IE2 tumors.

378 Apart from cDC2s, which represent the most frequent DC subset in our cohort and are strongly enriched in IE2, we were able to distinguish four other DC subsets previously described in 380 human breast tumors ${ }^{52}$ (Fig. 4D). Classical DCs type 1 (cluster cDC1), Langerhans cells (cluster LC) and plasmacytoid DCs (cluster pDC) were identified based on their high 382 expression of CLEC9A, CD20752 and IRF7/PLAC8/ IL3RA, respectively. A fifth DC subset showed elevated expression of $L A M P 3, F S C N 1$, and CCR7 and was identified as migratory

384 DCs (migDCs) as described recently in healthy thymus and various cancer types including breast $^{15,53-55}$. The migDC cluster displayed a particularly strong $T$ cell-suppression signature 386 compared to other DCs (Fig. 4E). We found that this cell type displayed the highest expression of CD274 (which encodes PD-L1) not only in the myeloid compartment but in the entire TIME

388 (Fig. 4F and Fig. S8A). It was also the main producer of the PDCD1LG2 mRNA, which encodes PD-L2, the second known PD-1 ligand. The migDCs also expressed IDO1, CD80, 390 and $C D 40$ and exhibited a unique cytokine expression profile with high counts of CCL17, CCL19, CCL22, and IL15, which were not detected in other myeloid cell subsets. In our IMC 392 dataset, we confirmed high levels of co-expression of PD-L1, IDO1, and CD40 proteins and CCL17 and CCL22 transcripts with the migDC marker LAMP3 (Fig. S8B, C). In summary, the 394 expression profile of migDCs suggests the potential to recruit immune cells and to strongly contribute to $T$ cell suppression.

396 Given that the abundance of migDCs did not differ between IE1 and IE2, we wanted to understand whether there was a difference in the DC transitional continuum. Different primary 398 DC subsets have the potential to become migDCs ${ }^{54,56}$. Slingshot trajectory inference analysis 
of our data implied the existence of a transitional state between cDC2s and migDCs, consistent with previous work suggesting that most migDCs in human tumors originate from cDC2 cells ${ }^{55,57}$; the cDC1 subset clustered apart (Fig. 4G). Using Monocle2 for trajectory

402 inference gave similar results (Fig. S8D). We observed distinct expression dynamics of the migDC markers such as CCL17, CCL22, and CCL19 along the CDC2-migDC transition (Fig. $404 \quad 4 \mathrm{H})$.

In summary, myeloid cells in exhausted and non-exhausted immune environments differed in 406 inflammatory cell states, and we observed evidence of crosstalk between myeloid cells and T cells. In addition, we have identified a LAMP3+ migratory DC subset that likely originates from cDC2 cells and displays a particularly high T cell-suppressive potential.

\section{Immune states are linked to distinct cell-to-cell communication patterns}

We then investigated systematically how cellular crosstalk differs in the IE1 and IE2 immune 412 environments by performing cell type interaction analysis using SingleCellSignalR, an algorithm that infers intercellular networks from single-cell transcriptomic data based on a 414 manually curated ligand-receptor database ${ }^{58}$. We first quantified the total number of predicted ligand-receptor interactions for each cell type pair across all samples (Fig. 5A, Fig. S9A). This

416 revealed many predicted interactions between fibroblasts and endothelial cells and myeloid cell auto-interactions. T cells, B cells, and plasma cells were generally predicted to have a 418 lower number of interactions, partly explicable by the overall fewer expressed genes in these cell types. Analysis with a second algorithm for inference of cellular crosstalk, CellPhoneDB,

420 confirmed these trends (Fig. S9B). To separate cell type-specific from ubiquitous interactions, we used the score coefficient of variation of the 100 top-scoring predicted ligand-receptor 422 interactions for each of the main cell type pairs to identify pair-specific interactions (Fig. S9C). We found that, for example, fibroblast-to-endothelial interactions were dominated by collagens

424 and integrin receptors, whereas specific myeloid-to-T cell crosstalk involved interactions of several cytokines with their cognate receptors.

426 We next compared predicted interactions in exhausted versus non-exhausted breast tumor immune environments. We applied SingleCellSignalR to data from each patient individually 428 (focusing on epithelial, myeloid, and T and NK cell interactions) and found a number of predicted ligand-receptor pairs enriched in either IE1 or IE2 tumors (Fig. 5B). As expected,

430 many predicted immunomodulatory interactions such as $C D 274$ with $P D C D 1$ (at the protein level, PD-L1 with PD-1), CD80 with CTLA-4, LGALS9 with HAVCR2 (at the protein level,

432 Galectin-9 with Tim-3), and PVR with TIGIT were enriched in IE1 tumors, with mRNAs encoding the ligands for PD-1 and CTLA-4 expressed at significantly higher levels only by

434 myeloid cells. IE1 tumors also had increased scores for chemotactic interactions, mainly mediated by CCL3, CCL4, CCL5, and CXCL9, suggesting the presence of a more dynamic 436 immune environment with increased recruitment of cells from outside the tumor. Furthermore, several predicted non-chemotactic cytokine interactions were enriched in IE1 tumors, some

438 activating (CSF-1 and IL15 with respective receptors), and others with more complex predicted functions (e.g., IL10 with its receptor). The only predicted cytokine interaction enriched in IE2

440 tumors was that of FLT3 ligand, a stimulator of DC growth ${ }^{59}$ and expressed by T and NK cells and its receptor on myeloid cells. Other interactions enriched in IE2 tumors included those of 
442 ERBB4 and of thrombospondin with their ligands. Performing a comparable analysis with CellPhoneDB yielded similar results (Fig. S9D).

444 To examine how specific myeloid cell subtypes and T and NK cell subtypes found in exhausted and non-exhausted environments may interact with each other, we repeated the interaction

446 analysis focusing only on these subtypes (Fig. 5C). For better biological interpretability, we first aggregated the identified clusters into larger metaclusters, each representing a specific

448 cellular subtype. All T and NK cell and myeloid metacluster pairs had between 300 and 600 predicted interactions, with TAM auto-interactions the most frequent. With the exception of

450 migDCs, cell types with many outgoing interactions (i.e., the cells express the ligand) also had many incoming interactions (i.e., the cells express the receptor). Predicted ligand-receptor

452 interactions that were specific for certain metacluster pairs (Fig. S9E) included the interaction between Tfh cell-specific IL21 and IL13 with respective receptors on myeloid clusters, the

454 interaction between CCL18 and CCR8 (on TAMs and $\mathrm{T}_{\text {regs, }}$, respectively) and that of CCL17 and CCL22 (expressed by migDCs and CDC2s) with CCR4 (expressed predominantly by $\mathrm{T}_{\text {regs }}$

456 and Tfh). As a next step, we wanted to predict which myeloid-derived ligands are the most closely linked to T cell exhaustion. For this purpose, we applied NicheNet, a method that does 458 not directly look at ligand-receptor interactions, but instead builds upon prior knowledge on signaling networks to predict which ligands of the sender cells (in our case, myeloid cells) are

460 most likely to have affected the expression of a set of known target genes (in our case, CD8+ $T$ cell exhaustion-related genes) in the receiver cells. The myeloid-derived ligand that was

462 identified by NicheNet to best predict expression of exhaustion-related genes was IL15, followed by IL1B and CXCL16 (Fig. 5D). This is consistent with the fact that the IL15-IL15

464 receptor interaction is enriched in IE1 tumors compared to IE2 tumors (Fig. 5B, S9D). The main producer of IL15 are migDCs (Fig. 4F), providing yet another link between migDCs and

466 T cell exhaustion.

In conclusion, ligand-receptor analysis provided a comprehensive quantitative map of 468 potential interactions across breast cancer TIMEs. We report an enrichment of $\mathrm{T}$ cellregulatory interactions and enhanced cytokine and chemokine signaling in IE1, especially from

470 myeloid cells to T and NK cells, compared to IE2, suggesting a balance of ongoing immune cell recruitment and regulation in exhausted environments.

\section{Spatial distribution of immune cells varies with exhaustion}

474 Ligand-receptor analysis can predict intercellular communication, but it is blind to an important requirement for cellular interaction: spatial proximity. Therefore, we sought to use our IMC 476 data to examine the spatial distribution of cell types and ask whether cells that are predicted to interact based on their ligand and receptor expression patterns are also physically close to each other in the tissue, and whether spatial patterns differ in IE1 and IE2.

We used pairwise neighborhood analysis to quantify relative avoidance or interaction for each cell type pair (accounting for cell type frequency) ${ }^{60}$ and found that fibroblasts and endothelial cells were strongly enriched in each other's neighborhood (Fig. 6A) (rectangle \#1), consistent with predicted ligand-receptor interactions between the two (Fig. 5A). Similarly, spatial analysis is consistent with predicted myeloid cell auto-interactions (rectangle \#2). As expected, tumor cell subtypes formed a spatial cluster (rectangle \#3) and tended to avoid other cell types. 
However, some tumor subtypes did show spatial proximity to other cell types: hypoxic and apoptotic tumor cells had a higher likelihood of neighbouring immune cells (rectangle \#4) and $\mathrm{PD}-1^{\text {high }} \mathrm{T}$ cells were more likely to border tumor cells than PD-1 ${ }^{\text {low }} \mathrm{T}$ cells (rectangle \#5). We 488 also observed spatial patterns within immune cell types. Myeloid cells, $\mathrm{T}_{\text {regs, }} \mathrm{CD} 4^{+} \mathrm{T}$ cells, and $\mathrm{CD}^{+} \mathrm{T}$ cells formed a spatial cluster (rectangle \#6), as did PD- $1^{\text {high }} / \mathrm{CD} 8^{+} \mathrm{T}$ cells, $\mathrm{PD}-$ $1^{\text {high }} / \mathrm{CD} 4^{+} \mathrm{T}$ cells, and migDCs (rectangle \#7).

Overall, we observed that PD-1 ${ }^{\text {high }} \mathrm{T}$ cells were significantly more likely to have at least one 492 migDC as their direct neighbor compared to PD-1 $1^{\text {low }}$ T cells (Fig. 6B-C). This is consistent with the predicted PD-1-PD-L1 interaction between myeloid and T cells identified in our ligand494 receptor analysis and with the finding that migDCs are the main producer of PD-L1 in our samples. Our data thus suggest direct engagement of PD-L1-expressing migDCs with PD-1496 expressing $T$ cells. To further examine exhaustion-associated spatial motifs, we quantified cell type composition of the immediate neighbourhood (10 closest neighbours) of different $T$ cell

498 subtypes in non-TLS images. This revealed that, on average, PD- $1^{\text {high }}$ T cells were surrounded by fewer myeloid cells and fibroblasts, but by more other PD-1 ${ }^{\text {high }} T$ cells, pDCs and migDCs, 500 when compared to PD-1 ${ }^{\text {low }}$ T cells and Tregs (Fig. 6D).

Pairwise neighbourhood analysis did not reveal major differences between the relative 502 interaction or avoidance of cell type pairs in IE1 compared to IE2 (data not shown), but IMC analysis showed a more than 5-fold higher proportion of cytokine-expressing cells in IE1 than 504 in IE2 tumors (Fig. S10A). We observed this increase for every cell type but especially for T and NK, SMA ${ }^{+}$stromal cells, and myeloid cells (Fig. S10B). In addition, a large proportion of 506 cytokine-expressing cells in IE1 but not IE2 tumors were part of a cytokine patch (defined as a spatial cluster of at least three cytokine-expressing cells directly neighboring each other) 508 (Fig. S10C). In order to identify the cell types surrounding these patches across the whole cohort, we defined cytokine milieus comprising all cells within a radius of $30 \mu \mathrm{m}$ from a given

510 cytokine patch and looked at the enrichment of cell types in these milieus compared to the overall tissue (Fig. 6E). We found that CD4 ${ }^{+} \mathrm{T}$ cells were enriched in CXCL9, CCL22 and

512 CXCL13 milieus, whereas CD8 ${ }^{+}$T cell enrichment was highest in CXCL9 and CXCL10 milieus (Fig. 6F, top). As expected, B cells were strongly enriched in CXCL13 milieus, and migDCs

514 were enriched with CCL17 and CCL22 milieus. Finally, myeloid cells were enriched in CCL18 milieus (Fig. 6F, bottom) and tumor cells were depleted across most cytokine milieus. Thus, 516 we conclude that spatial cytokine expression patterns are linked to cell type distribution and exhaustion state of the immune environment.

518 One of the most distinct structural elements found in solid tumors are TLS, which differ considerably from the surrounding tissue in terms of cell type frequency and spatial 520 distribution ${ }^{61}$. Our transcriptomics data showed higher frequency of Th cells and lower frequency of B cells in IE1 versus IE2 tumors, and was therefore contradictory for the likelihood 522 of TLS being present in these two groups (Fig. S4L). To directly examine TLS in both immune environments, we acquired immunofluorescence whole-slide scans of 13 samples from the 524 original Wagner et al. cohort and from 12 samples used in this study. We found that densely packed, mature TLS were more frequent in IE2-classified tumors, whereas immature TLS (see 526 methods for criteria, and Figure S2B) were more frequent in IE1-classified tumors (Fig. 6G). In IMC images, we found that most immune cell types were enriched in images containing 528 TLS compared to images that do not contain TLS and that there were only minor differences between immune cell frequencies in images that contained immature versus mature TLS (with 
530 the exception of B cells, which are highest in mature TLS as expected ${ }^{61}$ ) (Fig. S10D). Although the frequencies of $C X C L 13^{+}$T cells, which reportedly play a large role in TLS formation ${ }^{62}$, did

532 not differ significantly between images with immature versus mature TLS (Fig. S10E), the spatial distribution of these cells changed: Most $C X C L 13^{+} \mathrm{T}$ cells in images containing mature

534 TLS were part of a CXCL13-cytokine patch, the opposite was true for immature TLS images (Fig. 6H). Neighbourhood analysis on images containing mature or immature TLS further 536 revealed an enrichment of direct PD- $1^{\text {high }} \mathrm{T}$ cell-migDC interactions in these images compared with the full dataset (Fig. S10F).

538 In conclusion, spatial analysis was consistent with major predicted intercellular interaction axes and revealed that spatial clusters of cytokine-expressing cells were frequent in IE1 but

540 not IE2 tumors. In addition, our data suggest that migDC-mediated regulation of T cell activity occurs via direct interactions that take place preferentially in regions of mature and immature

542 TLS and that tumors with different immune environments differ in the spatial distribution of CXCL13 $13^{+}$cells.

544

\section{DISCUSSION}

546 Despite having a better prognosis than triple-negative and HER2 ${ }^{+}$tumors, the majority of patients who die of breast cancer have tumors of the Luminal subtype due to its high

548 incidence ${ }^{63}$. Immune-checkpoint therapy regimes for Luminal B tumors are currently being evaluated in clinical trials but have not been approved ${ }^{64}$. To better understand the responses

550 of breast cancer patients to immune modulators, we have performed detailed transcriptomic and spatial proteomic characterization of distinct breast tumor immune environments at single-

552 cell resolution. These analyses revealed systematic changes in the exhausted-like environment associated with chronic $T$ cell stimulation. We found that the presence of a PD-

$5541^{\text {high }}$ exhaustion-like $T$ cell phenotype was indicative of an inflamed immune environment with altered cytotoxic potential, cellular composition, and intercellular crosstalk.

556 scRNA-seq analysis revealed that in $\mathrm{CD}^{+} \mathrm{T}$ cells that express markers of exhaustion, hallmark genes of tumor-reactive $T$ cells are upregulated, consistent with results in other tumor 558 types $^{42}$. Strikingly, expression of MHC-I transcripts in tumor cells in IE2-classified tumors, which have few exhausted-like T cells, was substantially lower than in IE1-classified tumors.

560 MHC-I downregulation is one mechanism by which tumor cells escape immune surveillance ${ }^{65}$, and our data suggest that downregulation of this complex could explain the reduced tumor

562 reactivity of T cells in IE2 tumors. It has been shown that in a cohort of anti-PD1 treated breast tumor patients, low pre-treatment expression of $\mathrm{MHC}-\mathrm{I}$ on tumor cells was associated with 564 reduced $\mathrm{T}$ cell expansion on treatment ${ }^{15}$. Thus, we hypothesize that this form of immune evasion, observed in IE2 but not IE1 tumors, is not amenable to conventional immune

566 checkpoint therapy. Immune evasion of IE1 tumors, on the other hand, is linked to chronic stimulation and a progressively exhausted phenotype of tumor-reactive $T$ cells and may be

568 targetable by immune checkpoint blockade. Despite rigorous method validation and carefully considered cohort selection, our study has limitations: First, we did not study the third tumor-

570 immune group identified in Wagner et al.29 (representing 31\% of all tumors), as this group had generally low cell numbers after dissociation, a small immune proportion, and few $\mathrm{T}$ cells

572 compared to the other two groups. Second, the small size and low number of cells obtained 
from most breast tumors made additional experiments such as ex vivo validation of $T$ cell cytotoxicity impossible. Third, histological tumor grade is a co-variate that is not perfectly balanced in our cohort. However, we found that the characteristics that differed most 576 significantly between IE1 and IE2 showed no difference between low-grade and high-grade tumors in our cohort, and that accounting for grade in pseudobulk analysis did not substantially change the observed differential expression pattern.

Positive correlation of the exhausted-like $\mathrm{CD}^{+} \mathrm{T}$ cell proportion with that of an antigen580 experienced $\mathrm{CD} 4^{+} \mathrm{T}$ cell subset $(\mathrm{Tfh})$ indicates that the extent of tumor antigen exposure is similar for $\mathrm{CD}^{+}$and $\mathrm{CD} 4^{+} \mathrm{T}$ cells despite different antigen recognition mechanisms. Since 582 DCs may cross-present tumor antigens to both $\mathrm{CD}^{+}$and $\mathrm{CD} 4^{+} \mathrm{T}$ cells, this suggests that differential MHC-I expression by tumor cells is not the only reason for higher $\mathrm{T}$ cell stimulation 584 in IE1-classified tumors. Indeed, transcripts for a number of antigens such as CTAG2, MAGEA3, and MAGEA6, which are normally expressed in testis and are immunogenic when

586 aberrantly expressed in cancer tissue, are strongly expressed in a subset of IE1, but not IE2, samples (Supplementary Table S4). Although we do not have quantitative data on tumor 588 mutational burden or neoantigen load for our cohort, these factors have also been linked to T cell activation in breast and other cancer types ${ }^{62,66,67}$.

590 Contrary to the classical notion of $\mathrm{T}$ cell exhaustion but in line with findings in other cancer types ${ }^{13,14}$, T cells with characteristics of exhaustion in our cohort expressed markers of 592 proliferation. Differential gene expression analysis revealed that the cytotoxic effector potential of these exhausted-like cells was most probably altered but not abolished, and pseudotime

594 analysis suggested a decrease of TNF but an increase of GZMB and FASL expression during the progression to $T$ cell exhaustion, indicating a shift in the cytotoxic profile from classical 596 inflammatory cytokines to molecules mediating cytolytic effector functions. In contrast to reports from other tumor types ${ }^{13,14}$, we did not observe an increase of IFNG expression

598 associated with $\mathrm{T}$ cell exhaustion. The cytotoxic potential of IE1 environments was further enhanced by the presence of NKT cells, which highly expressed GNLY and GZMA; these cells

600 were almost absent from IE2 tumors. NKT cell accumulation in exhausted immune environments has not previously been reported, and its driving mechanism is unknown. In 602 addition to their cytotoxic role, we found that NKT cells might also contribute to myeloid cell activation and maturation via expression of the cytokine CSF1, providing a direct functional 604 link between the lymphoid and the myeloid blood cell lineages. The transcriptional profile of myeloid cells in IE1 environments revealed more inflammatory phenotypes and stronger signs

606 of surrounding tissue inflammation compared to IE2 environments. This increased tissue inflammation was accompanied by overexpression of matrix-remodeling metalloproteinases,

608 a phenotype linked to higher risk of metastasis ${ }^{51,68}$. Thus, although T and NK cells in exhausted immune environments retain cytolytic capacity, this does not appear to be sufficient to stop

610 disease progression and might even contribute to tumor invasiveness via inflammationinduced tissue remodeling. The strongly elevated number of cytokine-expressing cells in IE1

612 compared to IE2 tumors observed in IMC provides additional evidence for increased tissue inflammation in IE1. The tendency of these cells to form spatial clusters, surrounded by milieus

614 with cytokine-specific cell type composition, is also increased in IE1 tumors.

We identified a subset of myeloid cells as migDCs; this activated cell type has been described 616 only recently in healthy thymus and a number of cancer types including breast cancer ${ }^{14,15,53,57}$. The predicted role of these $L A M P 3^{+} \mathrm{DCs}$ in the TIME is complex: High levels of cytokine 
618 expression by these cells suggests a role in recruitment and activation of other immune cells, but migDCs also have a high T-cell suppressive potential, underlined by high expression of 620 IDO1 and CD274 (which encodes the PD-1 ligand PD-L1) and direct spatial interaction with PD- $1^{\text {high }} \mathrm{T}$ cells. Although PD-L1 expression in the TIME is associated with T cell exhaustion, 622 migDC frequency was comparable in IE1- and IE2-classified tumors. Pseudotime analysis indicated that most migDCs in our cohort originate from cDC2, a cell type significantly enriched

624 in IE2 environments, suggesting the presence of a large pool of potential migDC predecessors in non-exhausted environments that might counterbalance the expected overrepresentation 626 of migDCs in exhausted environments. In addition, the migratory phenotype predicted by the migDC transcriptional profile has been validated in vitro by Zhang et al., who also showed that 628 migration is lymph-node directed ${ }^{14}$. Thus, we speculate that migDCs might not accumulate at the tumor site but may migrate to the draining lymph node upon full maturation, providing an 630 explanation for the similar migDC frequencies observed in IE1 and IE2 tumors.

TLS are associated with favorable outcome in many cancer types; however, their prognostic 632 value in breast cancer is unclear ${ }^{69-73}$. Our transcriptional and protein-level analyses revealed that antigen-experienced $\mathrm{CD}^{+}$and $\mathrm{CD}^{+}{ }^{+} \mathrm{T}$ cells highly expressed the chemokine $\mathrm{CXCL} 13, \mathrm{a}$

634 B cell attractant essential for TLS formation ${ }^{62}$. Surprisingly, elevated CXCL13 expression in IE1 tumors did not translate into higher frequency of TLS. Instead, we observed more sites of

636 loosely accumulating B cells and $\mathrm{CXCL} 13^{+} \mathrm{T}$ cells that lacked the dense core typical for TLS structures and might represent either immature TLS or dissolving structures. Contrary to

638 mature TLS, where $\mathrm{CXCL} 13^{+} \mathrm{T}$ cells tended to occur in spatial clusters, $\mathrm{CXCL} 13^{+} \mathrm{T}$ cells in these immature sites often were spatially apart from each other. Occurrence of $\mathrm{CXCL} 13^{+} \mathrm{T}$

640 cells outside of immature or mature TLS was a defining characteristic of IE1 samples. This suggests that the immune microenvironment of IE1-classified tumors is more dynamic than

642 that of IE2-classified tumors, with continuing T cell-mediated signals to attract more immune cells.

644 Cell-to-cell interaction mapping revealed systematic enrichment of immunomodulatory, chemoattractive, and cytokine signaling in exhausted environments, corroborating the notion 646 that IE1 is a more dynamic immune environment than IE2. Although the focus of our analysis was on epithelial, myeloid, and T cells, taking advantage of our transcriptomic and spatial data 648 we identified interaction pairs across all cell types in IE1 and IE2 tumors, which provides a valuable resource for future studies. The endothelial-fibroblast axis with its demonstrated 650 spatial interaction and large number of predicted ligand-receptor pairs will be especially interesting to explore further.

652 PD-1 expression has previously been associated with poor prognosis in Luminal B tumors ${ }^{74}$, but because data on disease progression and survival is not yet available for our cohort, the 654 impact of IE classification on patient outcome remains to be confirmed. However, it has

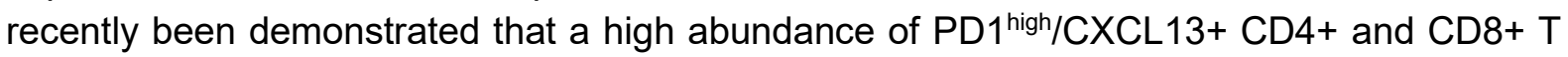

656 cells, as observed in IE1 tumors, is highly predictive for increased T cell expansion upon antiPD1 treatment across all breast cancer subtypes ${ }^{15}$ and positively influences response to anti-

658 PD-L1 treatment in triple-negative breast cancer. ${ }^{75}$ Irrespective of prognostic or predictive value, we have shown that IE1 and IE2 represent two distinct immune environments with 660 respect to tumor antigen presentation, $T$ cell phenotypes, cytotoxic potential, and cellular crosstalk, all of which are likely to affect immunotherapy response. PD-L1 is currently the main 662 marker used to stratify patients for immune checkpoint therapy in breast cancer ${ }^{76}$. However, 
our data suggest that PD-1, CXCL13, and MHC-I, possibly along with previously defined T cell exhaustion markers such as LAG-3 and Tim- $3^{29}$, are better able to distinguish these immune environments. Thus, we propose that these factors be considered for patient stratification in upcoming clinical trials.

\section{METHODS}

\section{Clinical samples}

670 Primary breast tumor resectates and health-related data were collected in collaboration with the Patient's Tumor Bank of Hope (PATH, Germany) at the breast cancer centers at St. 672 Johannes Hospital Dortmund and Institute of Pathology at Josefshaus (Germany) and the University Hospital Giessen and Marburg, Marburg site (Germany) after obtaining written

674 informed consent from patients. The majority of patient samples sequenced in this study (12 of 14) were originally collected and analyzed by suspension mass cytometry (CyTOF) by

676 Wagner et al. ${ }^{29}$. For each of these samples, we had access to an aliquot of viably frozen cells derived from the same cell suspension that was used in the original study. These samples

678 were previously classified as either TIG2 or TIG3 by Wagner et al. (corresponding to IE1 and IE2 in this study) based on immune cell subtype frequencies. The remaining two samples

680 (T_BB330 and T_BB338) were collected for this study, and pathological staging was performed by experienced pathologists as previously described ${ }^{29}$. All tissue and health-related 682 data were collected under approval of the Ethics Committee Zurich (\#2016-00215) and the faculty of medicine ethics committee at Friedrich-Wilhelms-University Bonn (\#255/06). Part of

684 each tumor was formalin-fixed and paraffin-embedded for the use in standard diagnostics and for analysis by IMC. For CyTOF and scRNA-seq analyses, a tissue sample of about $5 \times 5 \times 5$

$686 \mathrm{~mm}$ was taken prior to paraffin embedding. Thus, the tumor area used for standard diagnostics and IMC was spatially separate from the tumor area used for mass cytometry and SCRNA-

688 seq. For two out of 14 samples, IMC measurements were not possible due to unsuitable FFPE material or missing patient consent for FFPE-based analysis. Tumor subtypes in this study 690 were defined as follows: Luminal A (ER ${ }^{+}$and/or PR ${ }^{+}, \mathrm{HER2}-\mathrm{Ki}^{-67^{+}<20 \%}$ ), Luminal B (ER ${ }^{+}$ and/or PR ${ }^{+}, \mathrm{HER} 2^{-}, \mathrm{Ki}-67^{+} \geq 20 \%$ ), Luminal B-HER2 ${ }^{+}\left(\mathrm{ER}^{+}\right.$and/or $\mathrm{PR}^{+}, \mathrm{HER}^{+}$), HER2 ${ }^{+}$(ER',

$\left.692 \mathrm{PR}^{-}, \mathrm{HER}^{+}\right)$, and triple negative (ER-, PR-, HER2-). Two out of 14 patients had received neoadjuvant chemotherapy prior to tumor resection (Supplementary Information, Table S1 -

694 clinical data), but we did not observe any significant difference between tumors from treated and untreated patients in terms of immune cell type frequency or phenotypes. In the original 696 Wagner et al. patient cohort, neoadjuvantly treated patients were evenly split between the three major tumor-immune groups (TIGs) ${ }^{29}$.

\section{Tissue preparation}

700 After surgical resection, fresh tissue samples were immediately transferred to pre-cooled MACS Tissue Storage Solution (Miltenyi Biotec) and were shipped at $4{ }^{\circ} \mathrm{C}$. For suspension 702 mass cytometry, the tissue was processed as previously described ${ }^{29}$. In brief, the Tumor Dissociation Kit, human and the gentleMACS Dissociator (both Miltenyi Biotech) were used for tissue dissociation, followed by filtering of the single-cell suspension. Cells were stained 
for viability with $25 \mathrm{mM}$ cisplatin (Enzo Life Sciences) and subsequently fixed with $1.6 \%$ paraformaldehyde (Electron Miscroscopy Sciences) before storage at $-80^{\circ} \mathrm{C}$. For scRNA-seq, an aliquot of the single-cell suspension was taken prior to viability staining and fixation and

708 viable cells were frozen in RPMI-1640 cell culture medium (ThermoFisher) supplemented with $10 \%$ fetal bovine serum and 10\% DMSO (Sigma Aldrich) using a Mr. Frosty Freezing

710 Container (ThermoFisher). Viable cell aliquots were stored in liquid nitrogen.

\section{Suspension mass cytometry}

For the two samples not previously analyzed by suspension mass cytometry, we performed

714 mass tag cellular barcoding, antibody labeling and mass cytometry acquisition as previously described using the same antibody clones (immune-centered antibody panel only) ${ }^{29}$. Basic

716 data analysis including dimensionality reduction and clustering were performed as described and allowed allocation of each sample to one of the two distinct immune environments explored in this study (based on the frequency of exhausted T cell phenotypes).

\section{scRNA-seq sample preparation, data acquisition and pre-processing}

For scRNA-seq, viable cell suspensions were quick-thawed in a $37^{\circ} \mathrm{C}$ waterbath for $<1$ minute and progressively diluted by slow stepwise addition of RPMI $+10 \%$ fetal bovine serum until the volume was 10 times that of the initial volume. After a 10-min centrifugation step ( $300 \mathrm{~g}, 4$

$724{ }^{\circ} \mathrm{C}$ ), the supernatant was removed and the Dead Cell Removal Kit (Miltenyi Biotech) was used according to manufacturer's instructions to exclude dead and apoptotic cells. The resulting

726 live cell fraction was washed once with $0.04 \%$ bovine serum albumin (BSA) in PBS (Sigma Aldrich) and resuspended in the same buffer. Cell quality and cell count were assessed using

728 a Neubauer chamber (Electron Microscopy Sciences), and the concentration was adjusted to 800-1000 cells/ $\mu \mathrm{l}$. scRNA-seq libraries were generated using the Chromium Single Cell 3'

730 Library \& Gel Bead Kit v3 from 10x Genomics, aiming for 10,000 single cells per library. All libraries were sequenced on a NovaSEQ6000 System (Illumina). Following quality control, raw

732 sequencing reads were aligned to the human reference genome GRCh38 and reads per gene per cell were quantified using CellRanger (10x Genomics, v3.0.1) (Supplementary

734 Information, Table $\mathbf{S 2}$ - technical details of the sequencing runs).

\section{scRNA-seq clustering and cell type annotation}

The resulting gene-by-cell matrices from CellRanger were transformed into Seurat objects

738 (Seurat v3.0.2) ${ }^{77}$, and downstream analysis was conducted in R v3.6.1 unless otherwise stated. High-confidence doublets were removed using the DoubletFinder package with the pK

740 parameter optimized for each sample individually ${ }^{78}$. Subsequently, all Seurat objects were merged and filtered to exclude cells with $>7500$ or $<200$ expressed genes, with $>75000$ read

742 counts or with $>20 \%$ of reads mapping to mitochondrial RNA. The remaining cells were normalized and scaled using the sctransform wrapper in Seurat ${ }^{79}$. Sctransform also identifies

744 highly variable genes, which were then used to construct Principal Components (PCs). PCs 
covering the highest variance in the dataset were selected based on elbow plots and heatmaps and used as input for graph-based clustering. Clusters were calculated using the FindNeighbours and FindClusters functions with the resolution parameter set to 2 and

748 visualized using the Seurat implementation of the dimensional reduction algorithm UMAP ${ }^{80}$. Differential gene expression analysis was performed for the 61 resulting clusters using the

750 Seurat implementation of the statistical framework MAST ${ }^{81}$. The main cell types were annotated based on the expression of established marker genes (EPCAM/CDH1 for epithelial

752 cells, $C D 3 / C D 4 / C D 8 / N C R 1$ for the T and NK cell fraction, CD14/ITGAXIHLA-DRA for myeloid cells, PECAM1/VWF for endothelial cells, PDGFRB/FAP for fibroblasts, MS4A2 for mast cells

754 and basophils, MS4A1 for B cells, and lg-encoding transcripts for plasma cells) (Supplementary Information, Table S3 -full differential expression results and annotation

756 info for each cluster). Due to large transcriptional overlaps, T and NK cells could not be clearly distinguished at this level and were thus annotated as one cell type. No large-scale 758 batch effects derived from individual patients, sequencing runs or immune environments were found upon visual inspection of the UMAP. Separate clustering of the epithelial cell fractions 760 from individual patient samples is biologically expected and not a batch effect.

762 For detailed analysis of immune cell subtypes, 14 clusters identified as T and NK cells and 10 clusters identified as myeloid cells were separately subset from the full dataset. Both subsets

764 were re-normalized using sctransform while regressing out percentages of mitochondrial RNA and contaminating ambient RNA (specifically, percentages of keratin-encoding transcripts and

766 percentage of $M G P$ likely derived from apoptotic/ruptured epithelial cells) as confounding factors. As for the full dataset, PCs covering the highest variance in each subset were selected

768 and used for graph-based subclustering, which was performed at a range of resolutions between 0.1 and 1.5. The final resolution parameter resulting in stable subclusters at desired 770 granularity was selected using clustering tree analysis ${ }^{82}$ and was 1 for the T and NK cell subset (Fig. S4A) and 0.8 for the myeloid subset. Low-quality subclusters (one T and NK cell cluster

772 with high keratin counts, one T and NK cell cluster and one myeloid cluster with very low read counts, and one myeloid cluster with high mitochondrial percentage) were removed before

774 performing DE analysis. The most differentially expressed genes for each subcluster were used for manual cell subtype annotation (Table S3). Due to ambiguous CD4/CD8 776 classification of some T cell clusters, we annotated $C D 8^{+}, C D 4^{+}$, or mixed T cell clusters based on the ratio of $C D 8 A$ and $C D 8 B$ to $C D 4$ transcript counts (Fig. S4E).

778

\section{Pseudobulk analysis of scRNA-seq data}

780 For pseudobulk comparison of IE1- versus IE-2-classified samples, raw gene counts were summed over all cells per sample and normalized by library size for boxplot visualization. This

782 was done separately for the grouped T and NK cells, for the myeloid cells, and for the epithelial cells. Differentially expressed genes were identified using the exactTest for single-factor

784 experiments from the edgeR package (v3.26.5) ${ }^{83}$. For this, non-normalized gene-sum matrices were used as input, lowly expressed genes were filtered out (cutoff: $>30$ read counts in at least

7863 samples) and library normalization was performed using the calcNormFactors function. 
To account for tumor grade, a similar analysis was performed with tumor grade as a blocking factor in the model design. Due to the inability of the exactTest to handle complex experimental designs, a quasi-likelihood negative binomial generalized log-linear model (glmQLFTest) was used to determine differentially expressed genes for this analysis ${ }^{83}$.

Genes with log counts per million $<1.5$ in the higher-expressing sample group were excluded from further analysis (Supplementary Material, Table $\mathbf{S 4}$ - complete pseudobulk analysis results for T and NK cells, myeloid cells, and epithelial cells).

794

\section{Pseudotime analysis of scRNA-seq data}

796 Linear pseudotime ordering was performed for a subset of six $\mathrm{T}$ cell clusters (T-naive, Tcytotoxic-1 to -4 , and T-CD8-exhausted) using the Bayesian latent variable statistical

798 framework Ouija ${ }^{46}$ with 6000 iterations. To speed up calculations, only a random subsample of 800 cells per patient from this $T$ cell subset was used as input. A set of 23 putative marker 800 genes was used for pseudotime learning (Supplementary Material, Table S5 - gene lists and gene signatures) and individual cells were ranked by assigned pseudotime values for further

802 analysis. Mean pseudotimes for patient samples and clusters were calculated as the average pseudotime rank for all $\mathrm{T}$ cells of the respective sample/cluster that were included in Ouija 804 analysis.

806 To study the origin of migDCs, pseudotime analysis without predefined topology was performed on a subset of three myeloid clusters (cDC1, cDC2, and migDC) using Slingshot $808(\mathrm{v} 1.7 .3)^{84}$ and Monocle (v2.12) ${ }^{85}$. Slingshot pseudotime values were extracted for the cDC2migDC transition (excluding $\mathrm{CDC} 1$ ), cells were ranked by pseudotime and gene expression

810 along pseudotime rank was plotted using the rolling average over 11 cells (selected as a good granularity for visualizing the major trends in the plot, Fig. 4H). Mean DC pseudotime per

812 patient sample was calculated as the average pseudotime rank of all cDC2 and migDC cells in the sample (Fig. S8G).

814

\section{Identification of myeloid cell gene signatures}

816 In order to interpret gene expression patterns observed across myeloid cells in the context of defined functional states, we compiled four distinct gene signatures. To assemble the T cell-

818 attractive and the $\mathrm{T}$ cell-suppressive myeloid cell gene signatures we performed a broad literature survey and manually extracted genes reportedly involved in chemoattraction or

820 myeloid-mediated suppression of T cells (Supplementary Material, Table S5 - gene lists and gene signatures). Gene lists for the M1 and M2 signatures were adopted from Azizi et al. ${ }^{27}$.

822 Prior to summing up signature gene counts to calculate a score for each signature in each cell, individual gene counts were normalized by the total cellular gene count and log824 transformed to reduce the relative dominance of highly expressed genes. Given that we only observed positive signature score correlations in our analysis, we also assembled a random control gene signature and found no correlation with either the attractive or the suppressive $\mathrm{T}$ 
cell signature indicating that there were no experimental or computational biases (data not shown).

\section{Combined ligand-receptor analysis using scRNA-seq data}

832

\section{SingleCellSignalR}

To assess cell-to-cell communication, we first used the SingleCellSignalR package ${ }^{58}$. For

834 SingleCellSignalR analysis of main cell type interactions, we subsampled 10,000 cells for each of the cell types and calculated interaction scores for all ligand-receptor pairs over all cell type

836 pairs. Autocrine and paracrine interaction scores were calculated separately and then merged into a single matrix. An empirically selected ligand-receptor (LR) score threshold of 0.4 was 838 used to define and quantify "true" interaction pairs for each cell type pair as recommended ${ }^{60}$ (Fig. 5A, Fig. S9A). In order to extract the most relevant hits, we wanted to identify high-

840 scoring, cell type pair-specific interactions. To this end, the 100 highest-scoring ligandreceptor interactions for each cell type were extracted, followed by identification of the five 842 ligand-receptor pairs with the highest coefficient of variation between all cell type pairs (i.e., the five most specific interactions for the respective cell type pair) (Supplementary Material,

844 Table S6 - SingleCellSignalR output). Of these, pairs were manually selected for plotting based on solidity of the literature evidence and biological interpretability of the interaction (Fig.

846 5B). SingleCellSignalR analysis of the T and NK cell metacluster and myeloid cell metacluster interactions was performed in the same way and with the same cutoffs (Fig. 5C). In Fig. S9C,

848 all interactions involving LC, pDC, or T-mixed as well as myeloid-myeloid and T and NK-T and NK interactions were excluded for clarity.

850 To compare ligand-receptor interactions between IE1- and IE2-classified tumors, SingleCellSignalR analysis for epithelial cell, myeloid cell, and T and NK cell interactions was

852 performed for each patient sample separately. The difference between LR scores of IE1 and IE2 samples were assessed using Wilcoxon rank-sum testing for each ligand-receptor pair in

854 each cell type pair. Among all interactions with $p<0.05$ (not corrected for multiple hypthesis testing) and a mean LR score $>0.4$, the biologically most relevant and literature-backed 856 interactions were chosen for plotting (Fig. 5B). Complete results can be found in the Supplementary Material (Table S6 - SingleCelISignalR output).

858

CellPhoneDB

CellPhoneDB, another algorithm for ligand-receptor analysis ${ }^{86}$, was used to assess the 860 consistency of our SingleCellSignalR results. One major difference between the two algorithms is that CellPhoneDB defines the significance of ligand-receptor interactions based

862 on random permutation of cluster labels, leading to large $p$ values for interactions that are present in many different cluster pairs. To analyze and quantify the interactions in the main cell types, we subsampled 3,000 cells per cell type and ran CellPhoneDB in Python with 1,000 iterations (Supplementary Material, Table S7 - CellPhoneDB output). As for

866 SingleCellSignalR analysis, we compared ligand-receptor interactions between IE1 and IE2 tumors by running CellPhoneDB on each patient sample separately and testing for enrichment 
868 of ligand-receptor pairs in either of the two IEs. The results were in good agreement with the SingleCellSignalR findings (Fig. S9D).

870

NicheNet

To predict which myeloid-derived ligands are the most closely linked to $T$ cell exhaustion in

872 our dataset we used NicheNet, a method that combines single cell expression data with prior knowledge on signaling networks and predicts which sender cell ligands are most likely to

874 have affected the expression of a set of known target genes in the receiver cells ${ }^{87}$. For this analysis, we defined all myeloid cells as sender cells and all CD8+ and naïve T cell clusters

876 as receiver cells. The target gene set included all genes that were specifically upregulated in the T-CD8-exhausted cluster as determined by differential expression analysis. NicheNet 878 quantifies ligand activity by determining the Pearson correlation coefficient between a ligand's a priori target predictions and the observed transcriptional response, with higher ranking 880 ligands having a better ability to predict the target gene set compared to the background of expressed genes.

882

\section{Classification of CD8+ T cell infiltration status}

884 The CD8+ T cell infiltration status was independently assessed by a clinical pathologist based on the spatial distribution of CD8+ T cells on whole-slide H\&E and IHC stains as described

886 previously ${ }^{43}$. In brief, tumors were classified as (i) immune desert if there were no (or very few) CD8+ $\mathrm{T}$ cells in the intratumoral and stromal compartment or at the tumor margin, as (ii)

888 immune-excluded if CD8+ T cells were only found at the tumor margin or in the stromal compartment but not infiltrating the tumor mass, or as (iii) inflamed if CD8+ T cells had

890 infiltrated the tumor mass and were found in direct contact with tumor cells. Desert and immune-excluded phenotypes are generally classified as "immune-cold", while inflamed phenotypes are classified as "immune-hot".

\section{IMC panels}

For spatial analysis of the breast immune environment, we designed and validated two IMC 896 panels that included antibodies against canonical cell type markers and known immunoregulatory targets with a focus on markers differentially expressed in IE1 versus IE2

898 in our scRNA-seq pseudobulk analysis. The Protein Panel was an all-antibody panel, and the RNA Panel was an RNAscope panel that included 10 cytokine/chemokine-targeting mRNA

900 probes, one negative control mRNA probe $(D a p B)$, and one mRNA probe targeting MS4A1 (the gene encoding CD20). More detailed information can be found in Supplementary Table

902 S8.

IMC sample preparation and immunofluorescence whole-slide scans 
906 Twelve samples were analyzed by IMC. For each, two consecutive 4- $\mu$ m thick sections (for staining with two different panels) were cut from the respective FFPE tissue block and 908 processed on slides with a combination of immunofluorescence and IMC staining. Slides intended for staining with the Protein Panel were deparaffinized in HistoClear (Biosystems)

910 three times (10 min per incubation) before being rehydrated in a graded alcohol series (ethanol:deionized water 100:0, 90:10, 80:20, 70:30, 50:50, 0:100; 5-10 min each). Antigen

912 retrieval was performed in Tris-EDTA buffer $(\mathrm{pH} 9)$ at $95^{\circ} \mathrm{C}$ for $30 \mathrm{~min}$ in a NxGen decloaking chamber (Biocare Medical). After cool-down, slides were blocked with $3 \%$ BSA in TBS (20

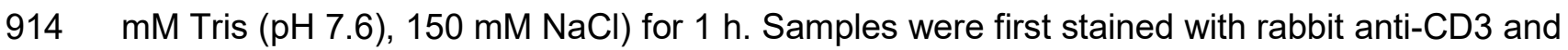
mouse anti-CD20 overnight at $4{ }^{\circ} \mathrm{C}$. Primary antibodies used at this stage were metal-tagged.

916 Slides were washed three time ( 5 min per wash) in TBS before staining with fluorescently labeled secondary antibodies (anti-rabbit-Alexa750 and anti-mouse-Alexa555) for $1 \mathrm{~h}$ at room

918 temperature. Slides were then stained with DAPI (1:500 in PBS), washed again three times (5 min per wash) in TBS and stained with the remaining metal-tagged antibodies of the Protein

920 Panel overnight at $4{ }^{\circ} \mathrm{C}$. The next day, slides were washed three times (5 min per wash) in TBS and stained with a final concentration of $0.5 \mu \mathrm{M}$ Cell ID Intercalator-Ir (Fluidigm) for 5 min

922 at room temperature. Slides were washed again in TBS (three times, $5 \mathrm{~min}$ per wash), dipped in deionized water, and dried with pressured air.

924 Slides to be stained with the RNA Panel were processed as previously described ${ }^{24,88}$. Antigen retrieval and RNA staining were performed with an RNAscope Fluorescence Multiplex

926 Reagent Kit (Advanced Cell Diagnostics) according to manufacturer's instructions. Detection oligonucleotides were metal-conjugated as described ${ }^{24}$ and used at a final concentration of 20

$928 \mathrm{nM}$. After the last wash step of the RNAscope protocol, slides were washed for 3 min in TBS and stained with metal-tagged rabbit anti-CD68, mouse anti-panCK, and mouse anti-E-

930 cadherin overnight at $4{ }^{\circ} \mathrm{C}$. Slides were then processed as described above with fluorescently labeled secondary antibody staining followed by DAPI staining, staining with the RNA Panel,

932 and iridium staining before the final washes and slide drying. Immediately after drying, an Axio Scan Z.1 (Zeiss) was used for multi-channel fluorescence whole-slide scanning of all sections.

934

\section{IMC region selection and data acquisition}

936 For IMC, four to six representative ROls were selected for each slide based on the immunofluorescence pre-scan. Immature and mature TLS regions were defined on the

938 immunofluorescence pre-scans as sites of B cell accumulation without a clear center or sites with dense round $B$ cell accumulation, respectively ${ }^{32}$. If present, one to five of these regions

940 were additionally imaged per sample. All ROls were $1 \mathrm{~mm} \times 1 \mathrm{~mm}$ and were manually aligned for each pair of consecutive tissue cuts to ensure the best possible overlap between images

942 from the two different panels. In total, 154 multiplexed images were acquired at $400 \mathrm{~Hz}$ using a Hyperion Imaging System (Fluidigm), and the raw data were pre-processed using

944 commercial software (Fluidigm) (Supplementary Material, Table S9 - ROI metadata). 
Images files were converted from the commercial format to OME-TIFF, and single cells were 948 segmented using a combination of ilastik $^{89}$ v.1.3.3 and CellProfiler ${ }^{90}$ v.3.1.9 as described in the workflow available at https://github.com/BodenmillerGroup/lmcSegmentationPipeline ${ }^{91}$.

950 Signal spillover between channels was compensated on the single-cell level using the $\mathrm{R}$ package CATALYST ${ }^{92}$ (v1.8.7), and high-dimensional averaged marker expression as well as 952 spatial features were extracted for each cell using CellProfiler ${ }^{91}$. Cells on the edges of each image were discarded. Single-cell objects extracted from tissue sections represent small

954 tissue slices that potentially contain overlapping cell fragments despite high-quality segmentation. Especially in densely packed regions, the extracted single-cell marker 956 expression may thus include some information originating from neighboring cells. To extract spatial cellular relationships, the circumference of each cell was expanded by 8 pixels $(8 \mu \mathrm{m})$ and overlapping cells were defined as neighbors.

\section{IMC analysis workflow and clustering}

IMC downstream analysis was conducted in R v.4.0.2. For each of the two panels, the single-

962 cell expression matrix was converted into a SingleCellExperiment object ${ }^{93}$ with the corresponding metadata attached, and a filter was applied to exclude cells with an area $<8$

964 pixels or $>600$ pixels. We used different data transformations for different downstream analysis steps as indicated in the figures: Single-cell marker expression was either (i) arcsinh-

966 transformed, scaled, and centered or (ii) 01-normalized for each marker using the $99^{\text {th }}$ percentile normalization to account for outliers. A multi-step graph-based clustering approach

968 based on the 01-normalized data was used to identify single-cell phenotypes. For each clustering step, a shared nearest neighbor graph was built using the scran $\mathrm{R}$ package

970 (v.1.16.0) $)^{94}$ followed by Louvain community detection as implemented in igraph (v.1.2.5 $)^{95}$. For each of the panels, the first clustering step was performed with a selected set of cell type

972 markers (see Suppl. Table S8), and each cluster was annotated as epithelial or non-epithelial based primarily on expression of panCK and E-Cadherin. Non-epithelial cell clusters were

974 pooled and subjected to a second clustering step with similar markers (excluding epithelial markers; see Fig. S2F and Fig. S3B for markers used) to identify immune and stromal cell

976 types in higher granularity. Annotation of stromal and immune cell subtypes was based on canonical marker expression. For the Protein Panel data only, T cell clusters were subsetted

978 and subjected to a third clustering step with T cell-specific markers such as PD-1 and ICOS (see Fig. S5B for markers used). To identify Ki-67+ T cells, an empirical cutoff of 0.7 was

980 chosen on Ki-67 arcsinh-transformed counts. In addition, epithelial cells from the Protein Panel that were proliferating, hypoxic, or apoptotic were identified using empirically defined cut-offs

982 for Ki-67 expression, Carbonic Anhydrase XI expression, and Cleaved Caspase/Cleaved PARP expression, respectively. The percentage of $\mathrm{Ki}-67^{+}$epithelial cells in each patient 984 sample as determined by IMC was strongly correlated to the percentage determined by immunohistochemistry in standard clinical diagnostics (Spearman's $\rho=0.77, p=0.0037$ ),

986 confirming sensible cut-off selection. We used the scater implementation ${ }^{96}$ of the dimensionality reduction algorithm UMAP for two-dimensional visualization of high-

988 dimensional single-cell data. For the full datasets, the algorithm was run on a random subsample of 10,000 cells per patient sample to avoid overcrowding of data points. For data 990 subsets, the algorithm was run on all cells. 
In the RNA Panel dataset, a binary cytokine expression status for each of the cytokines measured on mRNA level was assigned to every cell as previously described ${ }^{88}$. In brief, the difference between the respective cytokine signal and the signal of the negative control mRNA probe $(D a p B)$ was determined for each cell, $p$ values were calculated from the distribution of differences, and a threshold of $p<0.01$ after Benjamini-Hochberg correction was applied to 998 define cytokine-expressing cells.

\section{Cytokine patches and milieus}

Cytokine patches and cytokine milieus were defined as previously described ${ }^{88}$. In short, cytokine patches were defined as a minimum of three neighboring cells all expressing at least one cytokine (general cytokine patch) or all expressing the same cytokine (cytokine-specific

1004 patch). For this analysis, the maximum distance for two cells to be considered neighbors was $25 \mu \mathrm{m}$. To define cytokine milieus, the cytokine patch borders were extended by $30 \mu \mathrm{m}$ and 1006 all cells within this radius were included in the respective cytokine milieu. For each individual image, enrichment or depletion of a cell type $X$ in a milieu type $Y$ was calculated using a

1008 Fisher's exact test. The threshold for significance was $p<0.01$ and only images that contained both $X$ and $Y$ were used to quantify the percentage of images with significant enrichment or 1010 depletion of $\mathrm{X}$ in $\mathrm{Y}$.

\section{Functional gene signature validation by IMC}

To validate the T cell-attractive myeloid cell gene signatures, we used the RNA Panel dataset 1014 and split myeloid cells into two groups based on whether or not they expressed at least one of the measured T cell-attracting chemokines (CXCL9, CXCL10, CCL2, CCL17, CCL4, CCL5).

1016 Subsequently, the distance to the closest T cell was compared for chemokine-expressing and non-expressing myeloid cells. Of the genes used to construct the $T$ cell-suppressive myeloid

1018 cell gene signature, only antibodies targeting corresponding proteins IDO1 and PD-L1 were included in our IMC panels. For validation of this signature, we thus split myeloid cells of the 1020 protein panel IMC dataset into a "suppressive" group (high expression of PD-L1 and/or IDO1) and a "non-suppressive" group. For every myeloid cell, we then identified the 10 closest $\mathrm{T}$

1022 cells (excluding $T_{\text {regs }}$ ), calculated the proportion of PD- $1^{\text {high }} T$ cells among these, and compared this proportion for suppressive versus non-suppressive myeloid cells.

\section{Pairwise neighborhood analysis}

1026 The Protein Panel dataset was used for neighborhood analysis because it allowed a more fine-grained annotation of cellular subtypes than did the RNA Panel. To identify significantly enriched or depleted pairwise neighbor interactions between cell types, a two-sided 
permutation-test-based analysis with 1,000 permutations per image was performed as described previously ${ }^{60}$. The threshold for significant interactions was $p<0.01$.

1032

Image visualization

All pixel and single-cell level images shown in the figures were generated using the

1034 cytomapper R package (v.1.0.0)

\section{Whole-slide immunofluorescence screen for TLS classification}

To screen for mature TLS and immature TLS, we used FFPE slides from 13 additional patients 1038 included in the Wagner et al. cohort ${ }^{29}$ that were classified as either IE1 or IE2 (corresponding to TIG2 and TIG3 in Wagner et al.). Slide pre-processing and antigen retrieval were performed 1040 as described above for slides stained with the Protein Panel. After blocking with 3\% BSA in TBS, slides were stained with rat anti-CD3, rabbit anti-CD68, and mouse anti-CD20 overnight 1042 at $4{ }^{\circ} \mathrm{C}$ (Table S8). The next day, slides were washed in TBS (three times, 5 min per wash) and stained with fluorescently labeled secondary antibodies (Table S8) for $1 \mathrm{~h}$ at room

1044 temperature. Slides were washed again in TBS (three times, 5 min per wash), and a coverslip was mounted using a polyvinyl alcohol mounting medium. Fluorescent whole-slide images 1046 were acquired with an Axio Scan Z.1. Mature and immature TLS sites were defined as above (see IMC region selection and data acquisition section) and annotated in an IE-blinded 1048 manner.

\section{Data and code availability}

All data and code will be made available upon publication. RNA-seq data have been deposited 1052 in the ArrayExpress database at EMBL-EBI (https://www.ebi.ac.uk/arrayexpress) under accession number E-MTAB-10607. Imaging Mass Cytometry data have been deposited on 1054 Zenodo (DOI: 10.5281/zenodo.4911135) and will be made publicly accessible upon publication. 
1056

1058

1060

1062

1064

1066

1068

1070

1072

1074

1076

1078

1080

1082

1084

1086

1088

1090

1092

\section{REFERENCES}

1. Sosman, J. A. et al. Improved Survival with Ipilimumab in Patients with Metastatic Melanoma. N. Engl. J. Med. 363, 711-723 (2010).

2. Robert, C. et al. Durable Complete Response After Discontinuation of Pembrolizumab in Patients With Metastatic Melanoma. J. Clin. Oncol. 36, (2018).

3. Berghmans, T., Dingemans, A., Hendriks, L. E. L. \& Cadranel, J. Immunotherapy for nonsmall cell lung cancer : a new therapeutic algorithm. Eur. Respir. J. 55, (2020).

4. Wang, J. et al. Role of immune checkpoint inhibitor-based therapies for metastatic renal cell carcinoma in the fi rst-line setting : A Bayesian network analysis. EBioMedicine 47, 78-88 (2019).

5. Vonderheide, R. H., Domchek, S. M. \& Clark, A. S. Immunotherapy for breast cancer: What are we missing? Clin. Cancer Res. 23, 2640-2646 (2017).

6. Ali, H. R., Chlon, L., Pharoah, P. D. P., Markowetz, F. \& Caldas, C. Patterns of Immune Infiltration in Breast Cancer and Their Clinical Implications : A GeneExpression-Based Retrospective Study. PLoS Med. 13(12), 1-24 (2016).

7. Park, Y. H. et al. Chemotherapy induces dynamic immune responses in breast cancers that impact treatment outcome. Nat. Commun. 11, 1-14 (2020).

8. Denkert, C. et al. Tumour-infiltrating lymphocytes and prognosis in different subtypes of breast cancer: a pooled analysis of 3771 patients treated with neoadjuvant therapy. Lancet Oncol. 19, 40-50 (2018).

9. Kwapisz, D. Pembrolizumab and atezolizumab in triple-negative breast cancer. Cancer Immunol. Immunother. 1, (2020).

10. Raskov, H. Cytotoxic CD8 + T cells in cancer and cancer immunotherapy. Br. J. Cancer (2020) doi:10.1038/s41416-020-01048-4.

11. Matsushita, H., Hosoi, A., Ueha, S., Abe, J. \& Fujieda, N. Cytotoxic T Lymphocytes Block Tumor Growth Both by Lytic Activity and IFNg-Dependent Cell-Cycle Arrest. Cancer Immunol. Res. 3, 26-37 (2015).

12. Thommen, D. S. \& Schumacher, T. N. T Cell Dysfunction in Cancer. Cancer Cell 33, 547-562 (2018).

13. Li, H. et al. Dysfunctional CD8 T Cells Form a Proliferative, Dynamically Regulated Compartment within Human Melanoma. Cell 176, 1-15 (2018).

14. Zhang, L. et al. Lineage tracking reveals dynamic relationships of $\mathrm{T}$ cells in colorectal cancer. Nature 564, (2018).

15. Bassez, A. et al. A single-cell map of intratumoral changes during anti-PD1 treatment of patients with breast cancer. Nature Medicine (Springer US, 2021). doi:10.1038/s41591-021-01323-8.

16. Egelston, C. A. et al. Human breast tumor-infiltrating CD8+ T cells retain polyfunctionality despite PD-1 expression. Nat. Commun. 9, 1-11 (2018). 
1094 17. DeNardo, D. G. \& Ruffell, B. Macrophages as regulators of tumour immunity and immunotherapy. Nat. Rev. Immunol. 19, 369-382 (2019).

1096 18. Wculek, S. K. et al. Dendritic cells in cancer immunology and immunotherapy. Nat. Rev. Immunol. 20, 7-24 (2020).

1098 19. Murdoch, C., Muthana, M., Coffelt, S. B. \& Lewis, C. E. The role of myeloid cells in the promotion of tumour angiogenesis. Nat. Rev. Cancer 8, (2008).

$110020 . \quad$ Chen, W. et al. A multicenter study benchmarking single-cell samples. Nat. Biotechnol. doi:10.1038/s41587-020-00748-9.

1102 21. Ijsselsteijn, M. E., van der Breggen, R., Sarasqueta, A. F., Koning, F. \& de Miranda, N. F. C. C. A 40-marker panel for high dimensional characterization of cancer immune 1104 microenvironments by imaging mass cytometry. Front. Immunol. 10, 1-8 (2019).

22. Chevrier, S. et al. An Immune Atlas of Clear Cell Renal Cell Carcinoma. Cell 169, 736-749 (2017).

23. Giesen, C. et al. Highly multiplexed imaging of tumor tissues with subcellular resolution by mass cytometry. Nat. Methods 11, 417-422 (2014).

24. Schulz, D. et al. Simultaneous Multiplexed Imaging of mRNA and Proteins with

Subcellular Resolution in Breast Cancer Tissue Samples by Mass Cytometry. Cell Syst. 6, (2017).

25. Jackson, H. W. et al. The single-cell pathology landscape of breast cancer. Nature 578, 615-620 (2020).

26. Keren, L. et al. A Structured Tumor-Immune Microenvironment in Triple Negative Breast Cancer Revealed by Multiplexed Ion Beam imaging. Cell 174, 1373-1387 (2018).

27. Azizi, E. et al. Single-Cell Map of Diverse Immune Phenotypes in the Breast Tumor Microenvironment. Cell 174, 1-16 (2018).

28. Yuan, X., Wang, J., Huang, Y., Shangguan, D. \& Zhang, P. Single-Cell Profiling to Explore Immunological Heterogeneity of Tumor Microenvironment in Breast Cancer. Front. Immunol. 12, 1-7 (2021).

29. Wagner, J. et al. A Single-Cell Atlas of the Tumor and Immune Ecosystem of Human Breast Cancer. Cell 177, 1-16 (2019).

30. Deutsch, A., Feng, D., Pessin, J. E. \& Shinoda, K. The Impact of Single-Cell Genomics on Adipose Tissue Research. Int. J. Mol. Sci. 21, (2020).

31. Graham-Pole, J., Davie, M. \& Willoughby, M. L. N. Cryopreservation of human granulocytes in liquid nitrogen. J. Clin. Patholo 30, 758-762 (1977).

32. $\mathrm{Li}, \mathrm{Q}$. et al. Prognostic value of tertiary lymphoid structure and tumour infiltrating lymphocytes in oral squamous cell carcinoma. Int. J. Oral Sci. 12, 1-8 (2020). 
34. Nayar, R. et al. TCR signaling via Tec kinase ITK and interferon regulatory factor 4 (IRF4) regulates CD8+ T-cell differentiation. Proc. Natl. Acad. Sci. U. S. A. 109, (2012).

1136 35. Kurachi, M. et al. The transcription factor BATF operates as an essential differentiation checkpoint in early effector CD8 + T cells. Nat. Immunol. 15, 373-383 $1138 \quad$ (2014).

36. Scott, A. C. et al. TOX is a critical regulator of tumour-specific T cell differentiation. Nature 571, 270-274 (2019).

37. Sade-Feldman, M. et al. Defining T Cell States Associated with Response to Checkpoint Immunotherapy in Melanoma. Cell 175, 998-1013 (2018).

38. Schrum, A. G., Turka, L. A. \& Palmer, E. Surface T-cell antigen receptor expression and availability for long-term antigenic signaling. Immunol. Rev. 196, 7-24 (2003).

39. Paillard, F., Sterkers, G., Bismuth, G., Gomard, E. \& Vaquero, C. Lymphokine mRNA and $T$ cell multireceptor mRNA of the Ig super gene family are reciprocally modulated during human T cell activation. Eur. J. Immunol. 18, 1643-1646 (1988).

40. Savas, P. et al. Single-cell profiling of breast cancer T cells reveals a tissue-resident memory subset associated with improved prognosis. Nat. Med. 24, 986-993 (2018).

41. van der Leun, A. M., Thommen, D. S. \& Schumacher, T. N. CD8+ T cell states in human cancer: insights from single-cell analysis. Nat. Rev. Cancer 20, 218-232 (2020).

42. Duhen, T. et al. Co-expression of CD39 and CD103 identifies tumor-reactive CD8 T cells in human solid tumors. Nat. Commun. 9, (2018).

43. Sobottka, B., Moch, H. \& Varga, Z. Differential PD-1 / LAG-3 expression and immune phenotypes in metastatic sites of breast cancer. Breast Cancer Res. 23, 1-11 (2021).

44. Hornburg, M. et al. Single-cell dissection of cellular components and interactions shaping the tumor immune phenotypes in ovarian cancer. Cancer Cell 1-17 (2021) doi:10.1016/j.ccell.2021.04.004.

1160 45. Gu-Trantien, C. \& Willard-Gallo, K. Tumor-infiltrating follicular helper T cells: The new kids on the block. Oncoimmunology 2, 8-10 (2013).

1162 46. Campbell, K. R. \& Yau, C. A descriptive marker gene approach to single-cell pseudotime inference. Bioinformatics 35, 28-35 (2019).

1164 47. Terabe, M. \& Berzofsky, J. A. Tissue-specific roles of NKT cells in tumor immunity. Front. Immunol. 9, 1-11 (2018).

1166 48. Boulakirba, S. et al. IL-34 and CSF-1 display an equivalent macrophage differentiation ability but a different polarization potential. Sci. Rep. 8, 1-11 (2018).

1168 49. Chen, J. et al. CCL18 from Tumor-Associated Macrophages Promotes Breast Cancer Metastasis via PITPNM3. Cancer Cell 19, 541-555 (2011).

1170 50. Cui, W. et al. HBXIP upregulates CD46, CD55 and CD59 through ERK1/2/NF-jB signaling to protect breast cancer cells from complement attack. FEBS Lett. 586, 766- 
51. Merdad, A., Karim, S., Schulten, H. \& Dallol, A. Expression of Matrix Metalloproteinases (MMPs) in Primary Human Breast Cancer: MMP-9 as a Potential Biomarker for Cancer Invasion and Metastasis. Anticancer Res. 34, 1355-1366 1176 (2014).

1178

52. Hubert, M. et al. IFN-III is selectively produced by $\mathrm{CDC} 1$ and predicts good clinical outcome in breast cancer. Sci. Immunol. 5, eaav3942 (2020).

53. Park, J.-E. et al. A cell atlas of human thymic development defines T cell repertoire formation. Science 367, (2020).

54. Zhang, Q. et al. Landscape and Dynamics of Single Immune Cells in Hepatocellular

55. Maier, B. et al. A conserved dendritic-cell regulatory program limits antitumour immunity. Nature 580, (2020).

56. Cheng, S. et al. A pan-cancer single-cell transcriptional atlas of tumor infiltrating myeloid cells. Cell 184, 792-809.e23 (2021).

57. Qian, J. et al. A pan-cancer blueprint of the heterogeneous tumor microenvironment revealed by single-cell profiling. Cell Res. 30, 745-762 (2020).

58. Cabello-Aguilar, S. et al. SingleCellSignalR: inference of intercellular networks from single-cell transcriptomics. Nucleic Acids Res. 48, e55 (2020).

59. Karsunky, H., Merad, M., Cozzio, A., Weissman, I. L. \& Manz, M. G. Flt3 ligand regulates dendritic cell development from Flt3+ lymphoid and myeloid-committed progenitors to Flt3+ dendritic cells in vivo. J. Exp. Med. 198, 305-313 (2003).

60. Schapiro, D. et al. histoCAT: analysis of cell phenotypes and interactions in multiplex image cytometry data. Nat. Methods 14, (2017).

61. Colbeck, E. J., Ager, A., Gallimore, A. \& Jones, G. W. Tertiary lymphoid structures in cancer: Drivers of antitumor immunity, immunosuppression, or Bystander Sentinels in disease? Front. Immunol. 8, 1-18 (2017).

63. Valla, M. et al. Molecular subtypes of breast cancer: Long-term incidence trends and prognostic differences. Cancer Epidemiol. Biomarkers Prev. 25, 1625-1634 (2016).

64. Caroline, J., Stotz, L. \& Canguel, A. Clinical Data on Immunotherapy in Breast Cancer. Breast Care 15, 450-469 (2020).

65. Aptsiauri, N., Ruiz-Cabello, F. \& Garrido, F. The transition from HLA-I positive to HLAI negative primary tumors: the road to escape from T-cell responses. Curr. Opin. Immunol. 51, 123-132 (2018).

66. Zhang, X. et al. Breast cancer neoantigens can induce CD8+ T-cell responses and antitumor immunity. Cancer Immunol. Res. 5, 516-523 (2017). 
67. Ghorani, E. et al. The T cell differentiation landscape is shaped by tumour mutations in lung cancer. Nat. Cancer 1, 546-561 (2020).

68. Gonzalez-Avila, G. et al. Matrix metalloproteinases participation in the metastatic process and their diagnostic and therapeutic applications in cancer. Crit. Rev. Oncol. I Hematol. 137, 57-83 (2019).

69. Gu-Trantien, C. et al. CD4 + follicular helper T cell infiltration predicts breast cancer survival. J. Clin. Investig. invest. 123, 2873-2892 (2013).

70. Martinet, L. et al. Human solid tumors contain high endothelial venules: Association with T- and B-lymphocyte infiltration and favorable prognosis in breast cancer. Cancer Res. 71, 5678-5687 (2011).

71. Figenschau, S. L., Fismen, S., Fenton, K. A., Fenton, C. \& Mortensen, E. S. Tertiary lymphoid structures are associated with higher tumor grade in primary operable breast cancer patients. BMC Cancer 15, 1-11 (2015).

72. Lee, H. J. et al. Tertiary lymphoid structures: Prognostic significance and relationship with tumour-infiltrating lymphocytes in triple-negative breast cancer. J. Clin. Pathol. 69, 422-430 (2016).

73. Lee, M. et al. Presence of tertiary lymphoid structures determines the level of tumorinfiltrating lymphocytes in primary breast cancer and metastasis. Mod. Pathol. 32, 7080 (2019).

74. Muenst, S. et al. The presence of programmed death 1 (PD-1)-positive tumorinfiltrating lymphocytes is associated with poor prognosis in human breast cancer. Breast Cancer Res Treat 139, (2013).

75. Zhang, Y. et al. Single-cell analyses reveal key immune cell subsets associated with response to PD-L1 blockade in triple-negative breast cancer. Cancer Cell 1-16 (2021) doi:10.1016/j.ccell.2021.09.010.

76. Adams, S. et al. Current Landscape of Immunotherapy in Breast Cancer: A Review. JAMA Oncol. 5, 1205-1214 (2019).

77. Stuart, T. et al. Comprehensive Integration of Single-Cell Data. Cell 177, 18881902.e21 (2019).

78. McGinnis, C. S., Murrow, L. M. \& Gartner, Z. J. DoubletFinder: Doublet Detection in Single-Cell RNA Sequencing Data Using Artificial Nearest Neighbors. Cell Syst. 8, 329-337.e4 (2019).

79. Hafemeister, C. \& Satija, R. Normalization and variance stabilization of single-cell RNA-seq data using regularized negative binomial regression. Genome Biol. 20, 576827 (2019).

1246 80. Mclnnes, L., Healy, J., Saul, N. \& Großberger, L. UMAP: Uniform Manifold Approximation and Projection. J. Open Source Softw. 3, 861 (2018).

1248 81. Finak, G. et al. MAST: A flexible statistical framework for assessing transcriptional changes and characterizing heterogeneity in single-cell RNA sequencing data. 
82. Zappia, L. \& Oshlack, A. Clustering trees: a visualization for evaluating clusterings at multiple resolutions. Gigascience 7, 1-9 (2018).

1254

83. Robinson, M. D., McCarthy, D. J. \& Smyth, G. K. edgeR: A Bioconductor package for differential expression analysis of digital gene expression data. Bioinformatics 26, 139-140 (2009).

1256

84. Street, K. et al. Slingshot: cell lineage and pseudotime inference for single-cell transcriptomics. BMC Genomics 19, 1-16 (2018).

85. Qiu, X. et al. Single-cell mRNA quantification and differential analysis with Census. Nat. Methods 14, 309-315 (2017).

86. Efremova, M., Vento-Tormo, M., Teichmann, S. A. \& Vento-Tormo, R. CellPhoneDB: inferring cell-cell communication from combined expression of multi-subunit ligandreceptor complexes. Nat. Protoc. 15, 1484-1506 (2020).

87. Browaeys, R., Saelens, W. \& Saeys, Y. NicheNet: modeling intercellular communication by linking ligands to target genes. Nat. Methods doi:10.1038/s41592019-0667-5.

88. Hoch, T. et al. Multiplexed Imaging Mass Cytometry of Chemokine Milieus in Metastatic Melanoma Characterizes Features of Response to Immunotherapy.

89. Berg, S. et al. Ilastik: Interactive Machine Learning for (Bio)Image Analysis. Nat.

90. McQuin, C. et al. CellProfiler 3.0: Next-generation image processing for biology. PLoS Biol. 16, 1-17 (2018).

91. Zanotelli, V., Damond, N. \& Strotton, M. ImcSegmentationPipeline: A pixel classification based multiplexed image segmentation pipeline | Zenodo. https://zenodo.org/record/3841961.

92. Chevrier, S. et al. Compensation of Signal Spillover in Suspension and Imaging Mass Cytometry. Cell Syst. 6, 612-620.e5 (2018).

93. Amezquita, R. A. et al. Orchestrating single-cell analysis with Bioconductor. Nat. Methods 17, 137-145 (2020).

94. Lun, A. T. L., Mccarthy, D. J., Marioni, J. C. \& Mcdavid, A. A step-by-step workflow for low-level analysis of single-cell RNA-seq data with Bioconductor. F1000 Res. 5, (2016).

95. Csardi, G. \& Nepusz, T. The igraph software package for complex network research. InterJournal 1695 (2006).

96. McCarthy, D. J., Campbell, K. R., Lun, A. T. L. \& Wills, Q. F. Scater: pre-processing, quality control, normalization and visualization of single-cell RNA-seq data in R. Bioinformatics 33, 1179-1186 (2017). package for visualization of highly multiplexed imaging data. Bioinformatics $36,5706-$ 5708 (2020). 

Figures

Fig. 1
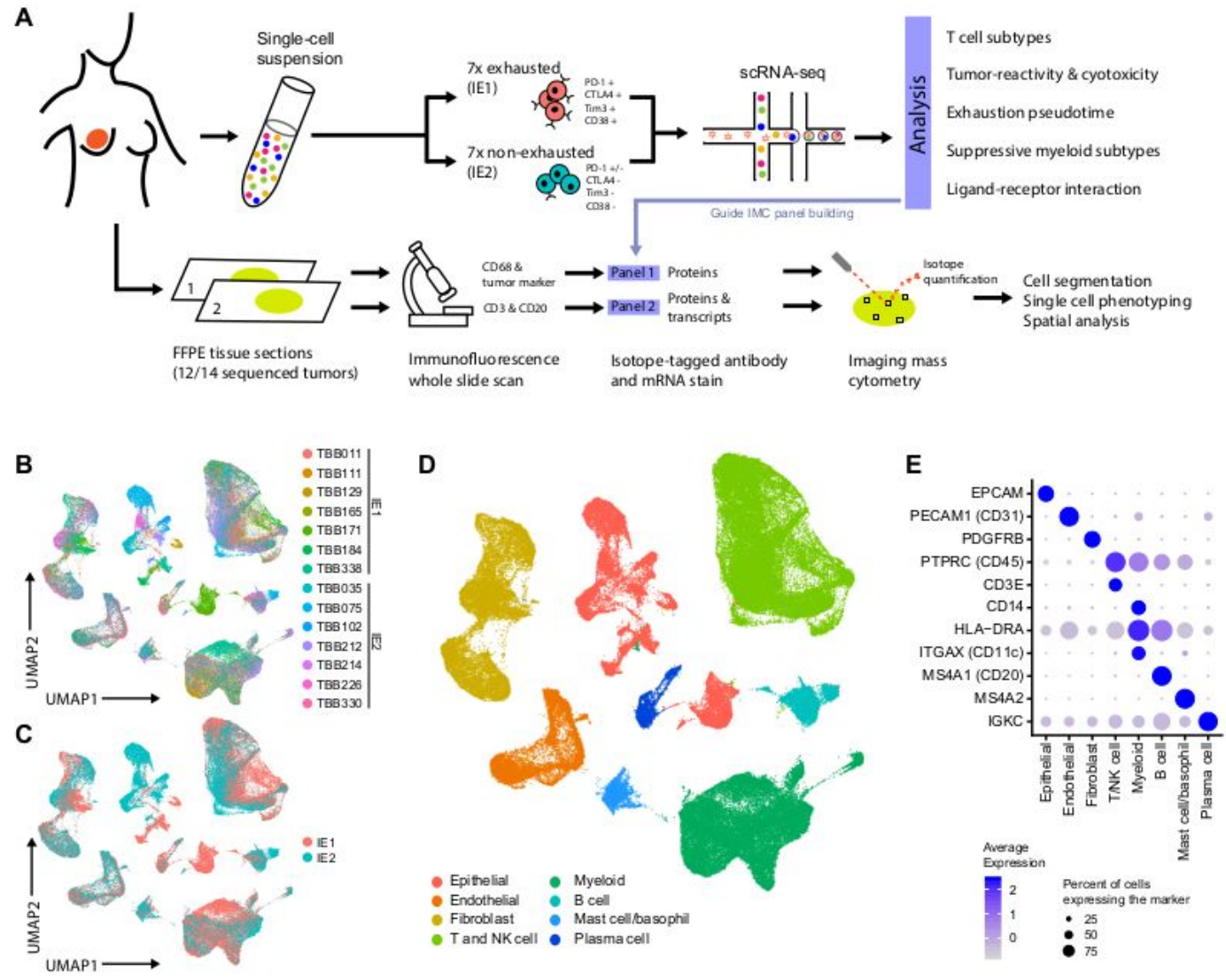

$\mathbf{F}$

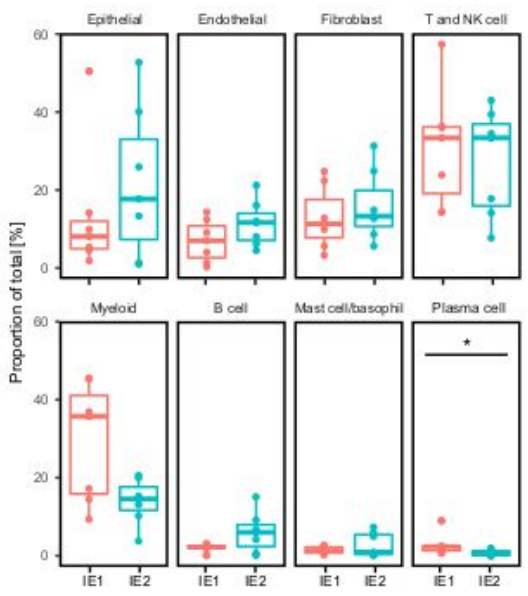

G
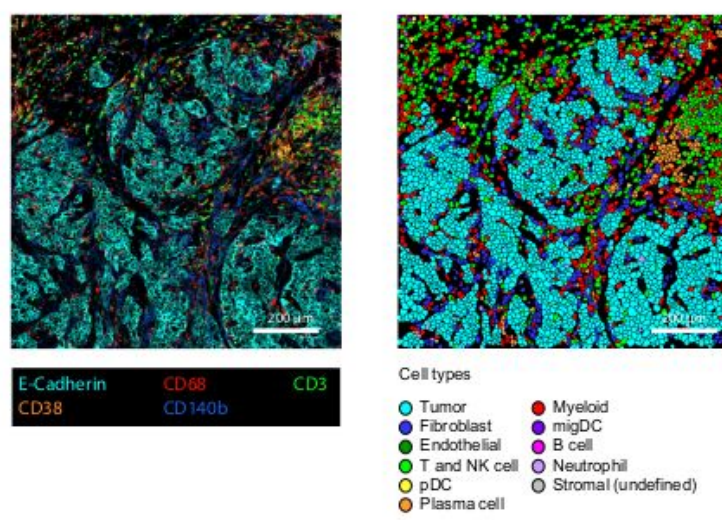

Figure 1

Transcriptomic and spatial proteomic analysis of breast tumor immune environments A. Sample selection and experimental approach. B. UMAP plot of scRNA-seq data from all 120,000 cells colored by patient. C. UMAP plot of scRNA-seq data colored by immune environment (IE). D. UMAP plot of scRNA- 
seq data colored by cell type. E. DotPlot showing transcript expression of main cell type markers in the indicated cell subsets. F. Proportion (\% of total cells) of main cell types in IE1 and IE2 tumors. Wilcoxon rank sum test was used for statistical analysis. ${ }^{*} \mathrm{p}<0.05$. Boxplot bodies show interquartile ranges (IQR), and whiskers extend to the largest and the smallest value within 1.5 times the IQR above the 75th percentile and below the 25th percentile, respectively. G. Exemplary IMC image showing staining patterns for the indicated markers. H. Single-cell masks for the IMC image displayed in panel $\mathrm{G}$ colored by cell type.

Fig. 2

A

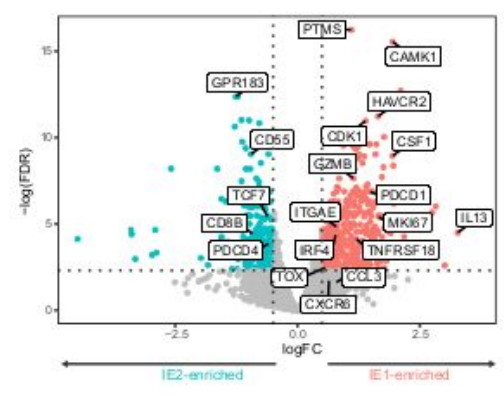

B

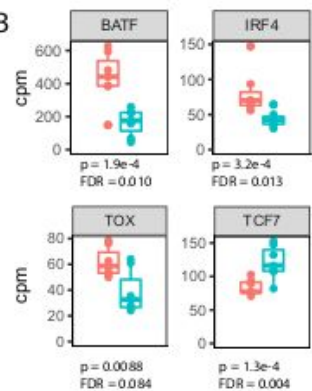

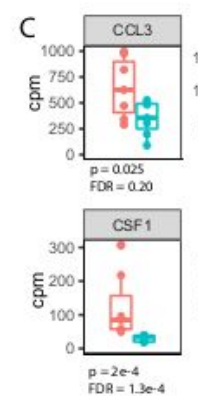

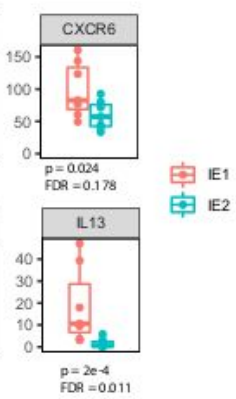

D
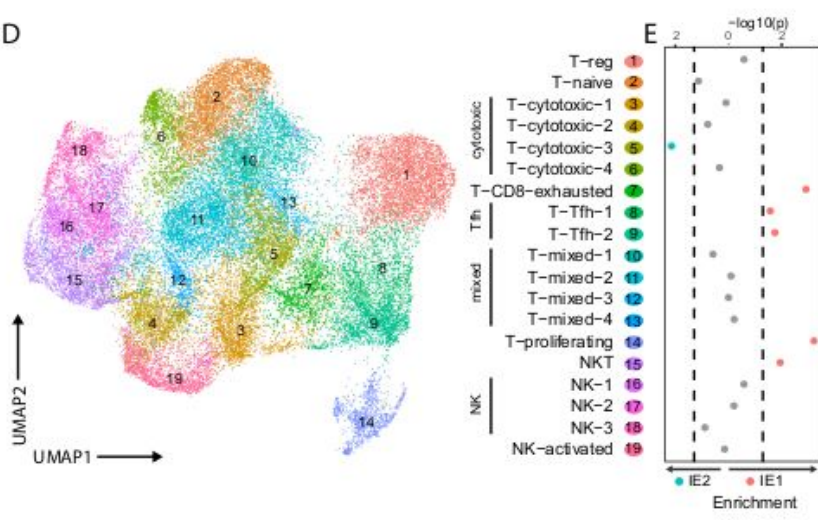

G
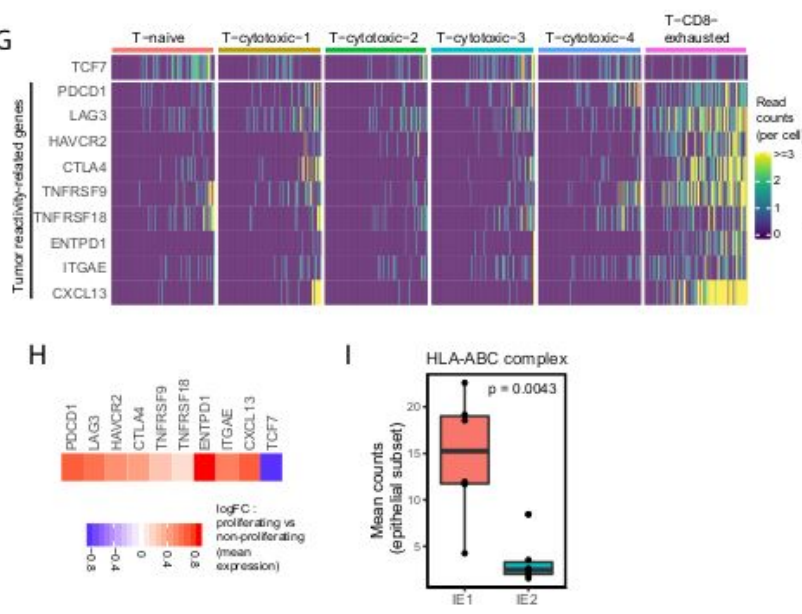

$\mathrm{F}$

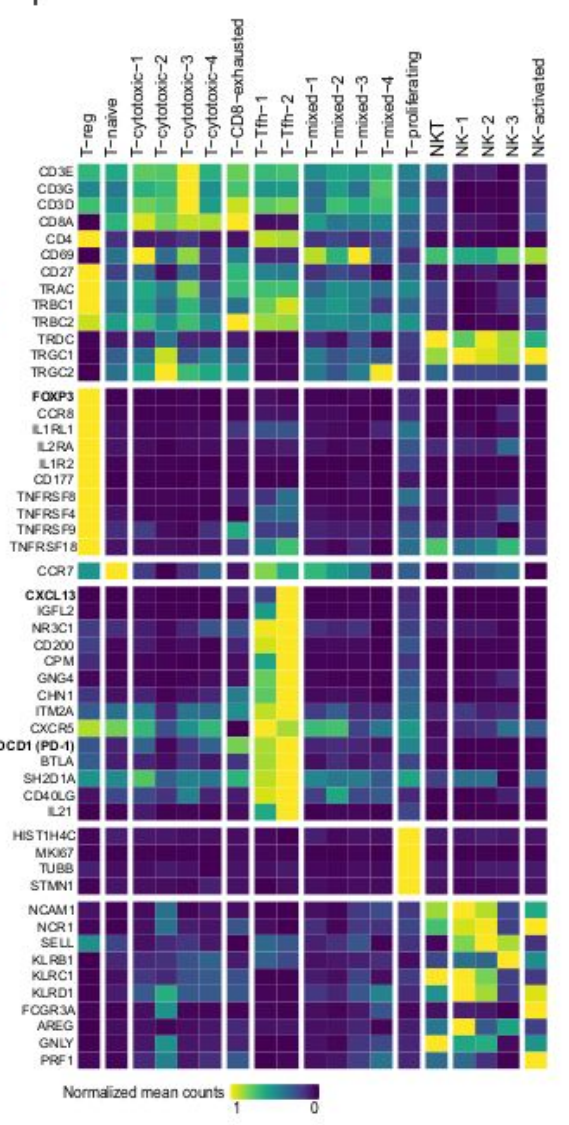

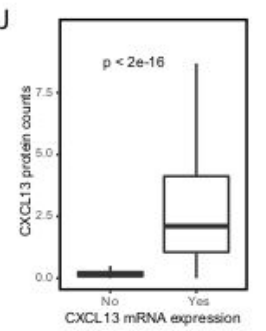
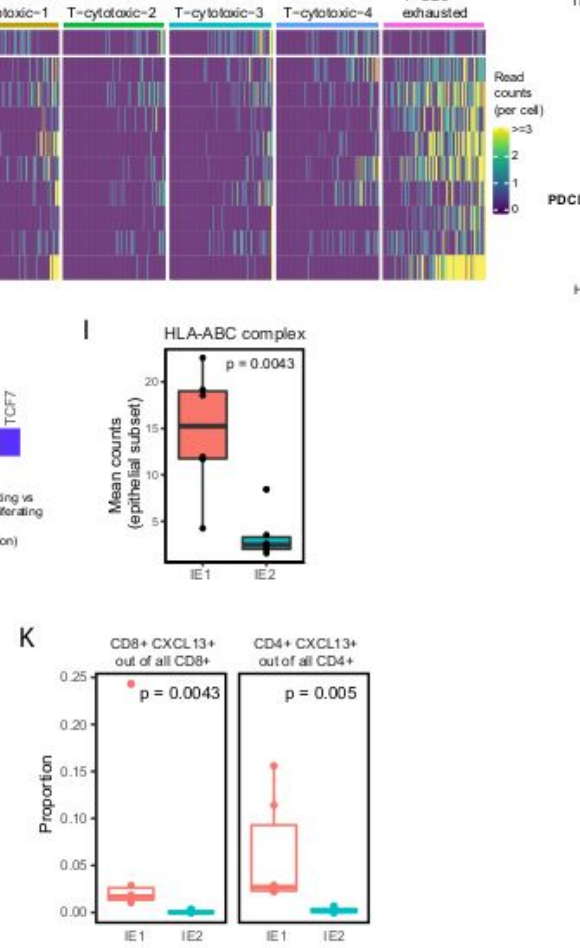


\section{Figure 2}

The T cell phenotypic landscape of exhausted and non-exhausted immune environments A. Volcano plot showing differential gene expression between T and NK cells of IE1 and IE2 samples in pseudobulk patient-averaged scRNA-seq data. Dashed lines indicate a false discovery rate (FDR) of 0.1 and a log2 fold change $(\log F C)$ of 0.5 . B. Boxplots comparing the $T$ and NK pseudobulk expression in counts per million (cpm) of selected transcription factors in IE1 and IE2 samples. C. Boxplots comparing the T and NK pseudobulk expression in cpm of selected cytokines and receptors in IE1 and IE2 samples. D. UMAP plot of scRNA-seq data from 36,000 T and NK cell cells colored by Seurat cluster, annotated with the indicated cell type labels. E. Enrichment of cluster frequencies, annotated by cell type, in IE1 or IE2 samples. Wilcoxon rank sum test was used for statistical analysis and dashed lines indicate a $p$ value of 0.05. F. Heatmap showing normalized average expression of selected marker genes for all $T$ and NK cell clusters. G. Single-cell count heatmap of selected genes associated with tumor-reactivity and/or exhaustion. For this analysis, 100 cells were randomly sampled from the naïve T cell cluster and from each CD8+ T cell cluster; columns represent single cells. H. Heatmap displaying the log-fold change of the mean expression of the indicated genes in proliferating versus non-proliferating $T$ and NK cells. I. Boxplot comparing the mean single-cell HLA-ABC expression in IMC data for the epithelial subsets of IE1 versus IE2 samples. J. Boxplot comparing mean CXCL13 protein counts between CXCL13-expressing and non-expressing T cells in IMC data. K. Boxplot comparing CXCL13high cell proportions out of all CD8+ T cells (left) and CD4+ T cells (right) between IMC IE1 and IE2 samples. Only non-TLS images were included and Wilcoxon rank sum test was used for statistical analysis. For scatterplots, Spearman correlation coefficient and $p$ value are indicated. For boxplots, Wilcoxon rank sum test was used for statistical analysis. Boxplot bodies show IQR, and whiskers extend to the largest and the smallest value lying within 1.5 times the IQR above the 75th percentile and below the 25th percentile, respectively.

\section{Figure 3}

Cytotoxic effector profiles differ between exhausted and non-exhausted immune environments A. Bar plot showing differential expression of the indicated transcripts in T and NK cells between IE1 and IE2 tumors (patient-averaged pseudobulk data). B. Heatmap showing normalized average single-cell expression of cytotoxic genes for all T and NK cell clusters. C. Pseudotime ordering of CD8+ T cells in all samples based on scRNA-seq data. Single cells are colored according to metacluster (bottom) and the corresponding density plot is displayed (top). D. Mean pseudotime scores for individual cell phenotype clusters. E. Mean pseudotime scores for individual samples colored by immune environment. F. Singlecell expression of the indicated cytotoxic genes along pseudotime. The analysis was done on scRNA-seq data from CD8+ T cells in all samples. Red line corresponds to locally estimated scatterplot smoothing (LOESS) curve. G. Average single-cell CSF1 expression in all T and NK cell clusters displayed as a bar chart (top) and in a normalized heatmap (bottom). 


\section{Figure 4}

Myeloid cell phenotypes in exhausted immune environments indicate inflammation and T cellsuppressive potential A. Volcano plot showing differential gene expression between myeloid cells of IE1 and IE2 samples in pseudobulk patient-averaged scRNA-seq data. Dashed lines indicate an FDR of 0.1 and a $\log F C$ of 0.5 . Genes are colored by functional group. B. UMAP plot of scRNA-seq data from 26,000 myeloid cells colored by Seurat cluster and annotated by cell type. C. Enrichment of cluster frequencies in IE1 and IE2 samples. Wilcoxon rank sum test was used for statistical analysis and dashed lines indicate a $p$ value of 0.05 . D. Heatmap showing normalized average single-cell expression of the top 10 differentially expressed genes for all myeloid cell clusters. Selected genes overexpressed in the respective cluster are indicated in the colored boxes. E. Scatterplot of the mean T cell-suppression score versus the mean T cellattraction score for all myeloid cell clusters. F. DotPlot showing expression of main migDC markers across all myeloid cell clusters. G. UMAP of CDC subsets and migDCs with Slingshot trajectories overlaid. H. Slingshot pseudotime ordering of single cells from the $\mathrm{CDC} 2$ and migDC subsets (top) and heatmap showing normalized expression of selected genes along pseudotime using the rolling average expression over 11 cells (bottom). Genes with log counts per million < 1.5 in EdgeR analysis were excluded for plots A-D. For scatterplots, Spearman correlation coefficient and $p$ value are indicated. 
Fig. 5

A

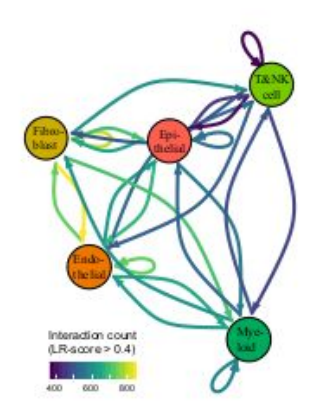

B

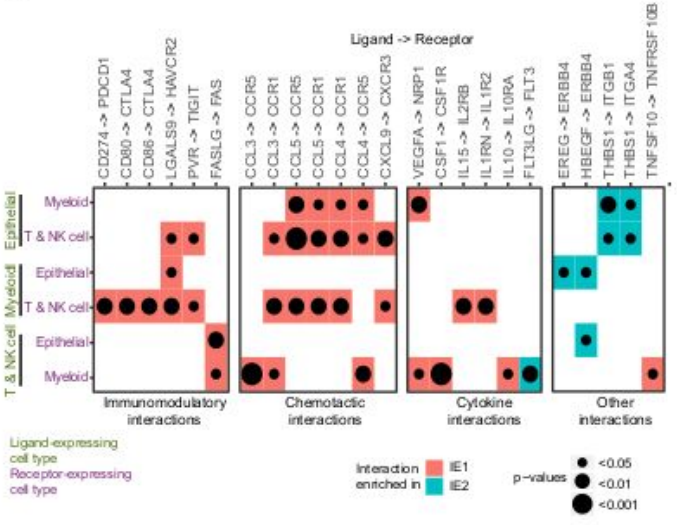

D

NicheNet priorifized myeloid ligands

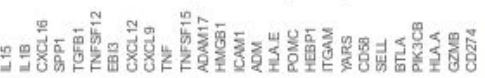
Ligand DIDIIIIIIIIIIII)

\section{C}
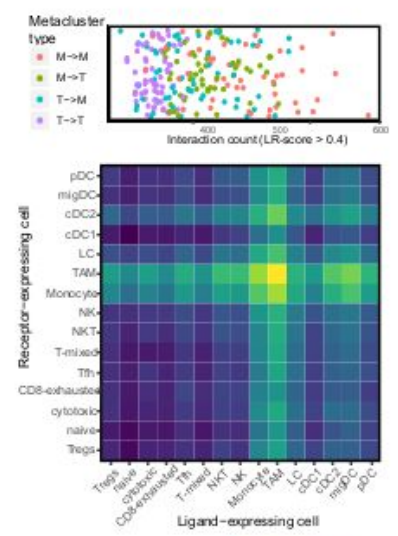

hreacton
(AR-sount

\section{Figure 5}

Ligand-receptor analysis predicts TIME-wide and exhaustion-specific cellular crosstalk A. Social graph depicting the number of interactions between the five most frequent cell types. B. Enrichment of selected ligand-receptor interactions in either IE1 or IE2 tumors for the given cell type pairs. Selections were made based on literature evidence and biological interpretability. The full list of enriched interaction pairs is in Supplementary Table 6 . White squares denote interactions with an enrichment $p$ value $>0.05$ or a mean 
LR score $<0.4$ in the given cell type pair. Wilcoxon rank sum test was used for statistical analysis. C. DotPlot (top) and heatmap (bottom) depicting the number of interactions between different myeloid and $\mathrm{T}$ and NK cell metaclusters. $\mathrm{M}$ indicates myeloid metacluster; $\mathrm{T}$ indicates $\mathrm{T}$ and NK cell metacluster. D. Heatmap showing the myeloid-derived ligands with the highest ability to affect exhaustion-related target gene expression as predicted by NicheNet.

\section{Figure 6}

IMC reveals cellular neighborhoods, cytokine milieus and tertiary lymphoid structures A. Heat map indicating significant pairwise cell type interaction or avoidance summarized across the two-sided permutation tests on the individual images of the Protein Panel dataset $(n=77$ images, 1,000 permutations each). Square color indicates the percentage of images with a significant cell-cell interaction or avoidance $(P<0.01)$, corrected for relative cell type frequency. Highlighted interactions (numbered boxes) indicate (1) fibroblast-endothelial interactions (2) myeloid autointeractions, (3) tumor compartment, (4) hypoxic/apoptotic tumor cell to immune cell interactions, (5) tumor to T cell subtype interactions, (6) main immune compartment, and (7) migDC-PD-1high T cell interaction. B. Single-cell masks for selected IMC images (top: mature TLS image, bottom: immature TLS image) colored by cell subtype. Only a subsection of each image is shown. C. Paired box plot comparing the percentage of PD1 high $T$ cells versus PD-1 low T cells that have at least one migDC as a direct neighbor. Each pair of dots represents a separate sample. A paired Wilcoxon rank sum test was used for statistical analysis. D. Heatmap displaying the average relative proportion of each celltype among the 10 nearest neighbours for each T cell subtype across all non-TLS images. E. Heatmap indicating significant relative enrichment or depletion of each cell type in the different cytokine milieus summarized across the Fisher's exact tests on all individual images of the RNA Panel dataset (total = 77 images; for each individual combination, only images containing the respective community and the respective cell type were included). F. Single-cell masks for selected IMC images with cell outline colored by the indicated cytokine community and cell body colored by cell type. G. Stacked barplots indicating the slide-wide TLS status for a cohort of 13 IE1 samples and 12 IE2 samples. H. Stacked barplots showing the proportions of CXCL13+ T cells that are part of a CXCL13-cytokine-cluster for non-TLS, immature TLS, and mature TLS images.

\section{Supplementary Files}

This is a list of supplementary files associated with this preprint. Click to download.

- Tables.zip

- TietscherSupplementalFiguresplusLegends.pdf 